\title{
Psicosomatica, PNEI e PNEIS spiegate attraverso la Teoria del Campo di Consapevolezza Unificato - TCCU
}

\section{Doi:https://doi.org/10.48274/IBI11}

\author{
Autori \\ Fabbroni R. (codice ORCID 0000-0001-5328-4412), \\ Sassola A. Biologo, Fisiopatologo, Chimico e Tecnologo del farmaco \\ Capello Lorenzo P. Medico Chirurgo, Omotossicologo Diplomato in Medicina Biologica e \\ Discipline Integrate
}

\begin{abstract}
La Teoria del Campo di Consapevolezza Unificato - TCCU, analizza lo stato di Benessere e quello di Malessere attraverso la Consapevolezza che l'essere umano possiede della vita che sta vivendo.

Si intende qui spiegare i processi psicologici di funzionamento legati alla salute di una persona in relazione ai campi di Energia-Informata che lo compongono e le loro interazioni con tutti gli altri campi esistenti in natura. Questo, secondo correlazioni e interconnessioni esistenti, di tipo Biofisico e Energetico-Informazionale, ci porterebbero alla conferma in cui lavisionedi corpo-mente e Spirito è frutto diinterconnessioni, che sono sostanzialmente uniti e sicuramente non separati.

In questo contesto diviene rilevante l'acquisizione e l'aumento della personale Consapevolezza che è un processo profondo, intimo e fondamentale per la Salute e che non è semplicemente uno stato di coscienza attiva ma è un Ente senziente mediatore tra i campi che compongono l'essere umano.

La Psicosomatica, la PNEI e la PNEI-S, rappresentano tre percorsi dello stato di benessere/malessere di una persona che vanno a sovrapporsi tra di loro in un processo evolutivo di analisi dello stato d'essere che inizia con la Psicosomatica ed evolve in PNEI e in ultima battuta in PNEI- S come vedremo.
\end{abstract}

La Teoria del Campo di Consapevolezza Unificato rappresenta una evoluzione/integrazione di questi tre percorsi.

\footnotetext{
Abstract

The Theory of the Unified Field of Consciousness - TCCU, analyzes the state of Well-being and the state of malaise through the Consciousness that the human being possesses of the life he is living.

Here we intend to explain the psychological processes of functioning related to the health of a person in relation to the fields of Unified Energy that compose it and their interactions with all other fields existing in nature. This, according to existing correlations and interconnections, of Biophysical and Energetic-Informational type, would lead us to the confirmation that the vision of body-mind and Spirit is the result of interconnections, which are substantially united and certainly not separated.
} 
In this context becomes relevant the acquisition and increase of personal Awareness that is a deep, intimate and fundamental process for Health and that is not simply a state of active consciousness but a sentient mediator between the fields that make up the human being.

Psychosomatics, PNEI and PNEI-S, represent three paths of the state of well-being / malaise of a person that overlap with each other in an evolutionary process of analysis of the state of being that begins with Psychosomatics and evolves in PNEI and ultimately in PNEI-S as we shall see.

The Unified Field of Consciousness Theory represents an evolution/integration of these three paths.

\section{Citazione per questo articolo}

Fabbroni R., Sassola A., Capello Lorenzo P., Psicosomatica, PNEI e PNEIS spiegate attraverso la Teoria del Campo di Consapevolezza Unificato - TCCU, Rivista Scienze Biofisiche (03/2021) DOI: $\underline{\text { https://doi.org/10.48274/IBI11 }}$

Psicosomatica (Roberto Fabbroni)

La medicina psicosomatica definisce il corpo e la mente come strettamente correlati.

Infatti il termine psicosomatico fu usato per la prima volta nel 1818 da Heinroth. Si trattava di un approccio olistico all'essere umano che trovava le sue origini già nell'antichità. Allo psicoanalista statunitense Alexander Lowen potremmo ricondurre la nascita della medicina psicosomatica, il quale sosteneva che ogni stato emotivo ha la sua sindrome fisiologica.

La Psicosomatica è la disciplina che studia quindi i legami tra i fattori psicologici e quelli fisiologici. La persona viene considerata come un'unità indivisibile in cui il disturbo si manifesta a livello organico come sintomo e a livello psicologico come disagio. La psicosomatica presta attenzione, dunque, non solo alla manifestazione fisiologica della malattia, ma anche all'aspetto emotivo che l'accompagna.

La sfera emotiva è quindi in grado di influenzare profondamente quella fisica determinando un dialogo che può esitare nella percezione di un disagio organico. Il corpo non può essere inteso come una macchina da riparare: esso esprime una complessità più grande che racchiude tutte le sfere che sono importanti per la vita di una persona.

Nel momento in cui si manifesta un disagio corporeo è necessario osservarlo prima da un punto di vista medico. La psicoterapia non può sostituirsi alla medicina ma la può completare e sostenere, aiutando una persona a rielaborare il disagio psicologico che si affianca a quello fisico. Talvolta la problematica fisica può addirittura non trovare riscontro in indagini organiche. In tali casi, è possibile supporre che la sfera emotiva incida sulla dimensione corporea esprimendo difficoltà che non sono mentalizzabili ed esplicitabili a parole.

È questo appunto, il caso delle malattie psicosomatiche. 
La somatizzazione è il meccanismo alla base dei disturbi psicosomatici e può essere definita come espressione di contenuti psichici in sintomi fisici, attraverso il coinvolgimento del sistema endocrino e immunitario.

Il vecchio concetto di malattia intesa come effetto di una causa è stato, dunque, pian piano sostituito con una visione multisistemica e multifattoriale bio-psico-sociale, secondo la quale ogni evento è conseguente all'intrecciarsi di molti fattori biologici/organici, psicologici e sociali, come ben vedremo con la Teoria del Campo di Consapevolezza Unificato.

Un esempio molto comune è la situazione in cui lo stress perdura nel tempo determinando una serie di reazioni che, a lungo andare, alterano le risposte organiche. Talvolta sono le strategie utilizzate per cercare di fronteggiare la causa dello stress (stressor) ad essere improprie e a favorire la comparsa di problemi organici ad esempio gastroenterici, cardiocircolatori, respiratori, urogenitali o dermatologici.

Una conseguenza di quanto appena esposto è la correlazione tra stress, emozioni e malattia coronarica.

Le malattie cardiovascolari sono la seconda causa di mortalità in tutti i paesi occidentali e hanno mostrato una generale tendenza all'aumento dall'inizio del secolo fino ad oggi.

Vi è motivo di ritenere che, accanto ai tradizionali fattori di rischio, determinanti di tipo emozionale, in gran parte collegabili a condizioni di stress cronico, siano responsabili di questo aumento di morbilità e di mortalità.

La ricerca degli ultimi anni ha dimostrato che stati emozionali come l'ansia, l'ostilità-aggressività o particolari 'stili comportamentali' (il tipo A) ${ }^{1}$ possono essere considerati come potenti fattori di rischio coronarico, soprattutto se associati ad una particolare concentrazione di eventi stressanti esistenziali. Questi fattori, oltre che nella patogenesi della malattia, giocano un ruolo rilevante anche nella prognosi e devono essere presi in considerazione in ogni programma sia preventivo che riabilitativo della malattia coronarica.

L'intervento del medico e dell'équipe terapeutica subisce una trasformazione radicale: passa quindi da un semplice controllo delle alterazioni biologiche tipiche della malattia ad un'azione multimodale, ad un controllo in grado di tenere costantemente presenti sia la personalità del paziente che le condizioni di stress emozionale preesistenti o successive all'episodio acuto.

In Italia invece le Malattie cardiovascolari sono la prima causa di morte ${ }^{2}$.

Le malattie cardiovascolari rappresentano ancora la principale causa di morte nel nostro Paese, in particolare muoiono più di 230 mila persone all'anno tra ischemie, infarti, malattie del cuore e cerebrovascolari (dati del 2017).

I disturbi psicosomatici si possono considerare quindi malattie vere e proprie che comportano danni a livello organico e che sono causate o aggravate da fattori emozionali.

\footnotetext{
${ }^{1}$ Friendman e Rosenman (1974) definiscono il comportamento di tipo A come uno specifico "sistema emozione-azione" esibito da coloro che si sentono coinvolti in una sfida incessante contro il tempo allo scopo di produrre sempre di più, esibendo un'aggressività razionalizzata, ma "liberamente fluttuante". Loro non attribuirono questo quadro a fattori di personalità, sia per non entrare in una tematica loro estranea sia per sottolineare il fatto che tali caratteristiche fossero reversibili e quindi assicurarsi la possibilità teorica di realizzare interventi utili ai fini terapeutici o preventivi.

${ }^{2} \mathrm{https}: / /$ www.salute.gov.it/rssp/paginaParagrafoRssp.jsp?sezione=situazione \& capitolo=quadro\&id=2645
} 
In particolare, rimarchiamo le situazioni di stress emotivo come, ansia patologica, paura costante o di forte preoccupazione che possono portare il fisico ad esprimere, sottoforma di campanello di allarme, un disagio più profondo.

I sintomi psicosomatici non derivano né da una condizione medica generale né dagli effetti diretti di una sostanza, ma dalla presenza di un disagio mentale.

I sintomi psicosomatici coinvolgono il sistema nervoso autonomo e forniscono una risposta vegetativa a situazioni di disagio psichico o di stress.

Le emozioni negative, come il risentimento, il rimpianto e la preoccupazione possono mantenere il sistema nervoso autonomo (sistema simpatico), in uno stato di eccitazione, nonché il corpo in una condizione di emergenza continua, a volte per un tempo più lungo di quello che l'organismo è in grado di sopportare.

I pensieri troppo angosciosi, quindi, possono mantenere il sistema nervoso autonomo in uno stato di attivazione persistente il quale può provocare dei danni agli organi più deboli.

A questo punto approfondiamo ulteriormente l'aspetto più problematico di tipo psicosomatico che è lo Stress e i disturbi cardiocircolatori che ne sono strettamente correlati, come ha dimostrato da tempo la ricerca scientifica.

\section{Che cos'è lo Stress?}

Formalmente si definisce stress una reazione che si manifesta quando una persona percepisce uno squilibrio tra le sollecitazioni ricevute e le risorse a disposizione. Si tratta, precisamente, di una sindrome generale di adattamento (SGA) atta a ristabilire un nuovo equilibrio interno (omeostasi) in seguito a fattori di stress (stressors).

Dal punto di vista fisiologico quando un tessuto è stressato significa che i potenziali elettrici delle membrane cellulari che lo costituiscono sono alterati.

In termini semplici possiamo paragonare ogni cellula alla batteria del nostro telefonino: quando si scarica, l'apparato emette segnali d'avvertimento, se non provvediamo alla ricarica il telefonino smette di funzionare.

Analogamente nelle nostre cellule con una bassa carica elettrica diminuisce anche la capacità di nutrirsi e di espellere le tossine. Durante questo processo sono lanciati segnali d'allarme che il nostro cervello interpreta come sintomi.

L'invecchiamento, i traumi, la presenza di virus, batteri e tossine o la formazione di radicali liberi possono costituire la causa dell'alterazione dei potenziali elettrici. Quando ciò accade il corpo perde la capacità d'autoriparazione e compare dolore, infiammazione, calo dell'efficienza e sintomi vari.

Lo stress (quello cronico, negativo) produce tra l'altro, infatti, il colesterolo (quello negativo, LDL), che a lungo andare danneggia le arterie. Ma il maggior problema delle malattie cardiocircolatorie oggi è stato accertato - non è tanto il cortisolo quanto una condizione emotiva alterata, nonché l'ansia, l'infiammazione e il disfunzionamento del sistema neurovegetativo. 
La presenza esagerata e cronica di rabbia, ad esempio, fa alzare, tra l'altro, la pressione arteriosa. La rabbia dovrebbe essere una "ultima spiaggia" quando, cioè, si vivono situazioni di ingiustizia forte, quando non ci si sente capiti e ascoltati, e quando non riescono altre modalità per modificare tutto ciò: la Forza calma, il Prendersi l'altro e portarselo dalla propria parte, la Dolcezza. Una diminuzione dell'eccessiva reattività emotiva è, dunque, di grande beneficio per il sistema cardiocircolatorio: l'emotività negativa troppo compressa finisce per esplodere in disturbi vari, danneggiando sia il cuore e sia la circolazione. Un altro nemico del funzionamento sano è la presenza dell'ansia. L'ansia è un sintomo collegato allo stress ma anche alla gamma dei disturbi d'ansia. L'ansia è connessa a un'attivazione di tutti i sistemi d'allarme dell'organismo umano, ma questo attivarsi non può durare a lungo perché produce squilibri e danni permanenti. All'allarme e all'attivazione dovrebbero seguire la disattivazione e l'allentamento; fasi che permettono il rigenerarsi dell'organismo a tutti i suoi livelli. Una buona regolazione neurovegetativa è altrettanto importante, ed è intimamente legata ai problemi di ansia, di stress, di attivazione esagerata e cronica. La condizione di vagotonia salvaguardia dagli spasmi arteriosi e genera un buon equilibrio biochimico dell'organismo. Ma allora possiamo fare qualcosa che sia da prevenzione dell'infarto e dell'angina pectoris?

Abbiamo strumenti per agire in modo benefico su queste problematiche?

Studiando lo stress e il funzionamento psico-corporeo dell'essere umano oggi abbiamo metodologie e tecniche che modificano nel profondo questi funzionamenti alterati, ripristinando condizioni normali meno negative per la salute, ripristinando un benessere di fondo. Ci sono tecniche mirate a riaprire o potenziare alcune ben determinate Esperienze di Base, esperienze fondamentali che, se consolidate positivamente in infanzia e adolescenza, danno luogo a Funzionamenti altrettanto fondamentali per la vita delle persone. E si tratta di tecniche che agiscono a livello multidimensionale, mente-corpo-energia. $\mathrm{Ci}$ sono tecniche che aiutano in modo specifico le Esperienze di Base più implicate nei problemi cardiocircolatori e più utili per aiutare la prevenzione e la salute, e cioè: la Calma, il Lasciare, l'Allentare il Controllo, la Forza calma, il Prendersi l'altro e portarselo dalla propria parte, il Benessere.

Noi possiamo diminuire gli eccessi di tensione muscolare in vari distretti del corpo, aiutando il drenaggio dei tessuti e migliorando la circolazione in genere, attraverso specifici movimenti e massaggi del profondo e tecniche bioenergetiche. Ad esempio la terapia del respiro è un altro punto nodale per la prevenzione dei disturbi del cuore. Ricostituire una respirazione diaframmatica spontanea profonda crea vagotonia, restituisce sollievo a tutto il sistema che regola gli equilibri delicati dell'organismo: pressione sanguigna diminuita, vasodilatazione invece che vasocostrizione, frequenza del battito cardiaco rallentata. In questo contesto portiamo l'attenzione in modo specifico sulla TB-Tecnica Bioenergetica del Metodo Summa Aurea ${ }^{\circledR}$ che si è dimostrata utile anche per diminuire i livelli di infiammazione (vedi bibliografia), con l'auspicabile dimostrazione futura della effettiva diminuzione delle citochine pro-infiammatorie. Il Metodo Summa Aurea ${ }^{\circledR}$, come vedremo nel dettaglio successivamente è Funzionale e valido non soltanto come strumento di prevenzione ma anche come coadiuvante nei processi di guarigione o ricerca del Benessere. 
Anche l'Analisi Bioenergetica ${ }^{3}$ che è una psicoterapia a mediazione corporea, rende molto bene l'idea di quanto fino a qui esposto.

Fu fondata in America negli anni cinquanta, come anticipato, da Alexander Lowen, brillante allievo di Wilhem Reich, da cui Lowen parte sistematizzando e ampliando alcuni dei principali concetti somatici del suo maestro.

Basandosi infatti sulla tesi dell'identità funzionale tra l'atteggiamento fisico di una persona e la struttura del suo io sottolinea come l'organismo umano funzioni come un tutto ed i versanti psichico e somatico sono solo funzioni apparentemente indipendenti ma di fatto strettamente correlate della funzione energetica globale.

Le tensioni muscolari croniche rappresentano la controparte fisica di conflitti psichici, attraverso di esse i conflitti si strutturano nel corpo sotto forma di restrizione del respiro e limitazione della motilità. Si tratta di tensioni che si sviluppano lentamente, attraverso esperienze traumatiche ripetute della prima infanzia, e si cronicizzato diventando parte inconsapevole della struttura corporea e del modo di essere di ognuno. Si perde pertanto del tutto la consapevolezza del loro significato, del perché si siano sviluppate e del come potersene liberare.

Nell'ambito dell'analisi bioenergetica ogni modello di tensione muscolare viene trattato a tre livelli:

1) la sua storia e origine nelle situazioni legate alla prima e seconda infanzia;

2) il suo significato attuale in rapporto al carattere dell'individuo;

3) il suo effetto sul funzionamento corporeo. Solo questa visione olistica può permettere quei cambiamenti della personalità che abbiano un valore duraturo.

Nella terapia il lavoro sugli aspetti fisici si combina sempre con la comprensione analitica del carattere, da cui appunto il nome di Analisi Bioenergetica.

"Per un paziente è altrettanto importante conoscere l'origine dei suoi conflitti quanto lo è acquistare consapevolezza di sé attraverso l'attività corporea. I due approcci devono essere sintonizzati tra loro perché la terapia sia efficace. Tutte le modalità psicoanalitiche e psicoterapeutiche vengono utilizzate nella terapia bioenergetica per favorire la comprensione e l'espressione di sé stessi. Questo include l'interpretazione dei sogni e l'elaborazione della situazione di transfert" (Lowen)

Tre tappe caratterizzano il processo terapeutico:

1) La consapevolezza di sé, sentire ogni parte del proprio corpo e i sentimenti che possono sorgere in esso dietro la maschera che ognuno si è costruito;

2) L'espressione di sé, se i sentimenti non trovano espressione vengono repressi e l'individuo perde il contatto con il Sè;

3) La padronanza di sé, l'individuo sa cosa sente, è in contatto con sé stesso e sa esprimersi in modo adeguato alla realtà.

${ }^{3}$ Tratto da: https://www.siab-online.it/home.aspx 
Il processo di cambiamento, quindi, coinvolge la persona in tutta la sua unità psicofisica, nella sua esperienza di essere vitale in quanto capace di respirare profondamente, sentire pienamente, ristabilire la naturale mobilità ed espressività dell'organismo, essere in contatto con la realtà del proprio modo di esistere e di rispondere emozionalmente.

Più una persona è emozionalmente disturbata più è lontana dal contatto con il proprio corpo, non è radicata nella propria realtà interna ed esterna, non ha "i piedi per terra", e, nella misura in cui non è radicata, ha una vita irreale, può crearsi illusioni sul proprio conto, può proiettare i propri problemi sugli altri.

Un'altra visione importante della Psicosomatica è quella di Walter Cannon, uno dei maggiori studiosi nel campo delle emozioni, padre e autore della Teoria Centrale dell'elaborazione emotiva.

Secondo Cannon le malattie psicosomatiche sono la conseguenza di un'inadeguata elaborazione di stimoli emotivi troppo intensi. La pressione emotiva mal gestita causa stress, cioè induce il corpo al mantenimento di uno stato di allerta troppo a lungo in attesa di fuggire o attaccare lo stimolo nocivo. Questa preparazione porta ad una attivazione fisiologica che deteriora gli organi più facilmente e prendendo di mira la nostra zona più vulnerabile. Se la mente ritiene di essere sotto attacco, il corpo si prepara, ma qualcuno dovrebbe avvisarli quando il pericolo cessa.

Il disturbo psicosomatico, riveste quindi un ruolo importante tra le malattie psichiche, poiché evidenzia come il corpo manifesti e comunichi inconsciamente uno stato di sofferenza mentale o di disagio psichico. La diretta scarica dell'emozione attraverso il corpo è una soluzione di difesa arcaico, meglio noto col nome di somatizzazione. La somatizzazione è il processo alla base del disturbo psicosomatico. Infatti, con tale termine si intende il meccanismo che permette di trasformare i processi psichici in somatici, coinvolgendo il sistema endocrino ed immunitario.

Solitamente questi meccanismi sono determinati dalla presenza di forte stress, da ansia patologica, da paura costante o da un forte disagio. Si attiva, così, il sistema nervoso autonomo, che a sua volta risponde con reazioni vegetative che portano alla manifestazione di problemi fisici, come:

- Disturbi gastrointestinali (colite ulcerosa, disbiosi intestinale, ulcera gastro-duodenale, reflusso gastroesofageo, rettocolite emorragica, coliche biliari, gastrite cronica, iperacidità gastrica, pilorospasmo, colon irritabile o spastico, stipsi, nausea e vomito, diarrea funzionale da emozione o esami)

- Disturbi respiratori (asma bronchiale, sindrome iperventilatoria, dispnea, singhiozzo, attacchi di panico)

- Disturbi cardiocircolatori (ipertensione arteriosa essenziale, tachicardia, aritmie, cardiopatia ischemica, coronopatie, cefalea emicranica, nevrosi cardiaca, algie precordiali)

- Disturbi urogenitali (dolori mestruali, disturbi minzionali, impotenza, infertilità, eiaculazione precoce o anorgasmia, enuresi)

- Disturbi cutanei (psoriasi, acne, eritema pudico, dermatite atopica, orticaria, prurito, neurodermatosi, iperidriosi, canizie, secchezza della cute e delle mucose)

- Disturbi nel sistema endocrino (ipertiroidismo o ipotiroidismo, ipoglicemia, diabete mellito)

- Disturbi nel sistema muscoloscheletrico (cefalea tensiva emicrania o mal di testa, stanchezza cronica, fibromialgia, crampi muscolari, mialgia, torcicollo, artrite, dolori al rachide cervicale e lombo-sacrale) 
- Disturbi dell'alimentazione (anoressia, bulimia, binge eating).

Tali manifestazioni corporee non sono prodotte intenzionalmente ma sono disagi reali. Questi sintomi organici possono portare ad un grado di sofferenza molto elevato in diverse aree del proprio funzionamento, come la vita affettiva, sociale, lavorativa e familiare. Quanto detto è frutto della Consapevolezza personale cioè della capacità di come affrontare l'evento o lo stato che stiamo vivendo.

L'analisi del rapporto tra psiche e corpo ci ha ben introdotti nelle reazioni Psicosomatiche introducendo le reazioni neuro-endocrine e somatiche di cui la PNEI e LA PNEI-S ne forniscono un quadro più esaustivo come ora andremo a vedere.

Concludendo possiamo dire che i condizionamenti derivanti dalla storia personale possono indurre, secondo la teoria dell'inibizione dell'azione, un'inibizione o un'iperattivazione dell'espressione comportamentale della persona che diventa disfunzionale e può comportare il disagio psichico fino al disturbo psichiatrico.

I comportamenti disfunzionali psicosomatici si manifestano nella percezione corporea-energeticaemotiva come blocchi. I blocchi più diffusi chiamati blocchi collettivi sono la chiusura dell'energia vitale, la chiusura del cuore e l'iperattivazione della mente controllante gli istinti e le emozioni.

Il riequilibrio psicosomatico dei comportamenti disfunzionali si attua con pratiche energetiche, manuali, emozionali e cognitive che consentono una regolazione dell'inibizione o dell'iperespressione.

Il riequilibrio è permesso dalla consapevolezza acquisita nel processo di rielaborazione del vissuto che coglie il senso dell'esperienza del disagio utilizzando l' informazione per evolvere e lasciar andare i condizionamenti. Ciò permette una riconnessione alla percezione di integrità e benessere globale definita Sé psicosomatico.

Riprenderemo più avanti il concetto di Consapevolezza e la sua rilevanza, oltre alle correlazioni che hanno le malattie cardiovascolari con la Teoria del Campo di Consapevolezza Unificato - TCCU.

Bibliografia (vale anche per PNEI-S)

- Angela Klopstech, Analisi bioenergetica e psicoterapia contemporanea: considerazioni dialogando con altri approcci $e$ con le neuroscienze (https://siabonline.it/media/58994/angela-klopstech.pdf)

- A.V.,Manuale di Analisi Bioenergetica, Franco Angeli ED., 2013 (Parte IV. Trattamento dei disturbi psicosomatici in analisi bioenergetica)

- Beffa, Nicole - Cantoreggi, Jessica (2015) L'implicazione del massaggio nella presa a carico fisioterapica in pazienti affetti da Depressione Maggiore. Bachelor thesis, Scuola universitaria professionale della Svizzera italiana (SUPSI)

- Nava Tiziana, Terapia manuale del paziente fibromialgico, DOI: https://doi.org/10.36017/jahc1908-010

- Arnot R. (2000). The Biology of Success. Sidney: Hodder.

- Barchas JD. and Friedman DX. (1963). Brain amines: Response to physiological stress. Biochemical Pharmacology 12:1232-1235. 
- Brown BS. and van Huss W. (1973). Exercise and rat brain catecholamines. Journal of Applied Physiology 30:664-669.

- Brown BS., Payne T., Kim C., Moore G., Krebs P. and Martin W. (1979). Chronic response of rat brain norepinephrine and serotonin levels to endurance training. Journal of Applied Physiology 46:19-23.

- Byrne A. \& Byrne DG. (1993). The effect of exercise on depression, anxiety and other mood states: A review. Journal of Psychosomatic Research 37 (No.6): 565-574.

- Dishman RK. (1986). Mental health. In: V.Seefeldt (Ed.) Physical activity and wellbeing (pp. 304-341). American Alliance for Health, Physical Education, Recreation and Dance; Reston VA.

- Dishman RK, Renner KJ, Youngstedt SD, RelgleTG, Bunnell BN, Burke KA, Yoo HS, Mougey EH and Meyerhof JL (1997) Activity wheel running reduces escape latency and alters brain monoamine levels after footshock. Brain Research Bulletin 42:399-406.

- Gordon R, Spector S, Sjoerdsma A and Udenfrlend S (1966) Increased synthesis or norepinephrine and epinephrine In the intact rat during exercise and exposure to cold. Journal of Pharmacology and Experimental Therapeutics 153:440-447.

- Grossman A \& Moretti A (1986) Opioid peptides and their relationships to hormonal changes during acute exercise. In: G. Benzi, L. Packer \& N. Slliprandi (Eds.) Biochemical aspects of physical exercise (pp. 375-386). Elsevier; Amsterdam.

- HarberVJ and Sutton JR (1984) Endorphins and exercise. Sports Medicine 1:154-171.

- Janal MN.Colt EWD, Clark WC and Glusman M (1984) Pain sensitivity, mood, and plasma endocrine levels in man following long distance running: effect of naloxone. Pain 19:1325 .

- Lowen A (1975) Bloenergetlcs. The Betrayal of the Body. Harper \& Row; New York.

- Lowen A and Lowen L (1977) The Way to Vibrant Health. Harper \& Row; New York.

- Lowen A (1972) Depression and the Body. Harper \& Row; New York.

- Lowen A (1958) The Language of the Body. Gruñe\& Stratton; New York.

- Lowen A (1980) Fear of Life. Harper \& Row; New York.

- Ostman I and Nyback H (1976) Adaptive changes in central and peripheral noradrenergic neurons in rats, following chronic exercise. Neuroscience 1:41-47.

- PertCB (1997) Molecules of Emotion. Simon \& Schuster; London.

- Salmon P (2001) Effects of physical exercise on anxiety, depression, and sensitivity to stress: a unifying theory. Clinical psychology Review 21 (no. 1): 33-61.

- Thoren P, Floras JS, Hoffman P and Seals DR (1990) Endorphins and exercise: physiological mechanisms and clinical implications. Medicine and Science in Sports and Exercise 22:417-428.

- Turner EE, Rejeski WJ and Brawley LR (1997) Psychosocial benefits of physical activity are influenced by the social environment. Journal of Sport and Exercise Psychology 19:119-130.

- Alexander Gerda: Eutonia (Paidos, 1991)

- Alexander Gerda: Le Corps Retrouvé par l'Eutonie (Tchou, Paris 1977, 1981)

- Brand Ulrich: Eutonia. La ginnastica per educare il corpo ai movimenti naturali (Red, 1995) 
- Validazione Scientifiche ed effetti fisiologici e psicologici delle pratiche di consapevolezza psicosomatica, (www.villaggioglobale.eu/documenti/gaia/validazioni_scientifiche_2014.pdf)

\section{Dalla PNEI alla PNEI CLINICA (Dr. Sassola Andrea)}

La PNEI o Psico-Neuro-Endocrino-Immunologia è comunemente conosciuta come la scienza integrata che studia l'attività concertata dei tre sistemi integrativi della Vita biologica dell'Essere Umano: il sistema nervoso, il sistema endocrino e il sistema immunitario.

Seppure tale disciplina sia nata nel secolo scorso con chiaro intento unificativo scientifico, è noto come nel tempo sia stata invece oggetto di progressive divisioni intestine, tanto da dover assistere alla configurazione di orientamenti anche opposti tra loro.

In linea generale ciò che accomuna le diverse correnti di pensiero generatesi da essa, così come le numerose discipline mediche e para-mediche integrate, è il riconoscimento di un'influenza della vita psicologica sul corpo fisico, nota per altro all'uomo già da millenni. Quel che di fondamentale viene per contro a mancare nella quasi totalità dei differenti approcci diagnostico-curativi è la conoscenza e talvolta persino l'interesse verso le dinamiche chimiche neuro-endocrine fondative su cui si fonda in verità l'unica PNEI, attraverso le quali ogni sorta di vissuto psicologico o spirituale giunge ad influenzare l'attività del sistema immunitario e in ultima analisi la condizione di salute del corpo fisico.

Nel migliore dei casi è oggi infatti possibile imbattersi in orientamenti medico-scientifici contemplanti il solo stress, il dolore o la depressione, condizioni a loro volta associabili esclusivamente a una chimica neuro-endocrino-immunitaria riferibile alla sola vita inconscia, e che pertanto escludono a priori lo studio di molecole psichedelico-espansive della coscienza, le quali oltre alla mediazione del piacere, consentono al corpo biologico di espletare fondamentali funzioni autorigenerative.

La conoscenza di composti psichedelici endogeni e esogeni, alla luce delle più moderne acquisizioni in materia di chimica biologica umana e vegetale, risulta dunque strettamente vincolante anche al solo fine di poter parlare di PNEI, la quale ultima può essere dunque intesa e a sua volta definita secondo tre livelli, analogici a tre stadi progressivi della coscienza stessa.

Un primo livello consente di interpretare la PNEI svolgendone semplicemente l'acronimo, definendola dunque quale scienza integrante le competenze di tre discipline fondamentali: la NeuroPsichiatria, l'Endocrinologia e l'Immunologia.

Il secondo livello concede in aggiunta di ri-conoscerla quale disciplina scientifica impegnata nello studio della chimica delle emozioni e degli stati di coscienza, intesi nella rispettiva distinzione psicochimica, e nell'indagine di come essa rifletta le proprie dinamiche sul corpo biologico, attraverso l'attività decisiva del sistema immunitario, il quale ultimo sancisce il verificarsi di condizioni di salute o malattia e in sintesi di vita o di morte.

In questo frangente diviene però vincolante il riconoscimento prioritario di una duplice origine della PNEI, la quale da un lato presenta per ovvie ragioni radici storiche scientifico-laboratoriali oggi divenute clinica, emerse già dalla prima metà del secolo scorso, dall'altra rivela un profondo fondamento filosofico-esistenziale nella rivoluzione psichedelica avvenuta nella seconda metà del novecento.

Fu proprio grazie ai movimenti giovanili di quegli anni che l'umanità d'occidente o meglio già in quel 
tempo "proto-occidentalizzata", ri-scoprì cosa nota da secoli all'antica sapienza, propria di ogni civiltà del globo terrestre, ovvero la capacità dell'Essere Umano di poter accedere a stati espansionali della coscienza.

Ancor oggi ciò che invece si presenta come amara conferma o rinnovato presagio, è l'interesse unilaterale per stati oppiaceo-benzodiazepinici di riduzione della coscienza stessa, limitante in sostanza l'indagine degli infiniti livelli diametralmente opposti che trascendono il razionale, concedendo all'uomo e alla donna l'accesso a una dimensione psichedelica archetipica, di fatto oggi esclusivamente sottoposta a un unico interesse giudicante, inquinato da precetti morali contrabbandati come tutela universale.

$\mathrm{Ne}$ consegue quanto di più paradossale, vale a dire l'associazione univoca e pubblica di molecole psicotrope potenzialmente rigenerative e fondamentali per la Vita, a vicende drammatiche di carattere sociale, le quali, seppur vere quanto spiegabili come frutto mortifero di una conoscenza biochimica nulla, non fanno altro che confermare un interesse feticistico verso una condizione anestetizzante che tende alla morte e che tra gli altri comprende stati sub-coscenziali di coma o pre-coma, da parte di una scienza etico-morale riconoscibile come non più autonoma nell'interpretazione di dati oggettivi. Avvenne però storicamente che chimici di fama internazionale quali Albert Hoffman e Alexander Shulgin, la cui vita scientifica fu inseparabile da quella della moglie Ann, annunciarono al globo la chimica delle molecole psichedeliche, il primo a partire dalla scoperta per serendipità degli effetti dell'LSD (1) a cui fece seguire lo studio e l'analisi di fitocomplessi psicotropi naturali, il secondo attraverso lo sviluppo e la caratterizzazione di infiniti composti psichedelici di carattere feniletilamminico e triptaminico $(2,3)$.

Furono poi autori più noti come il famigerato psicologo Timothy Leary, considerato profeta della "psicologia psichedelica" (4) a contribuire alla diffusione di massa di tali sensazionali scoperte, valutate poi esclusivamente sotto un profilo di tossicità sociale da parte di una società incosciente, nel timore dichiarato di deliri allucinatori omicio-suicidari e nel terrore occulto di una transizione evolutiva a Società Cosciente.

Lo stesso Dott. Leary, seppur nei modi teatrali stigmatizzati dallo stesso Hoffman con cui entrò in contatto diretto, seppe però straordinariamente intuire come il compito dello psicologo o dello psichiatra fosse quello di indagare la mediazione neurochimica degli stati di coscienza.

In realtà, anticipandolo storicamente di ben oltre mezzo secolo, Sigmund Freud, padre della psicanalisi, precognizzò nel 1895 in "Progetto di una Psicologia scientifica", "L'intenzione di fornire una psicologia che sia una scienza naturale; di rappresentare i processi psichici come stati di singole particelle materiali determinati quantitativamente e indagare i loro fondamenti neurochimici", intuizione questa che trova assoluta conferma nella moderna PNEI, rappresentandone in sintesi uno tra gli intenti fondativi di ricerca.

Per comprendere scientificamente una tale riflessione è però d'obbligo ri-conoscere ciò che nel corpo umano è scientifica evidenza, ossia quelle strutture biologicamente preposte alla percezione del Piacere, all'espansione della coscienza e all'auto rigenerazione, prima tra tutte l'epifisi o ghiandola pineale, patrimoniodi conoscenza della stessa endocrinologia ma ancor prima di culture millenarie sopravvissute tra oriente e occidente.

L'Universo nel trasporsi in biologia umana segue un percorso chemo-dipendente mediato dalla ghiandola pineale quale trasduttore neurochimico (5) in grado di orientare i sistemi biologici integrati in relazione alle condizioni energetiche universali, le quali sono sintetizzabili nella condizione archetipica di alternanza tra luce e buio, caratteristica del ciclo circadiano. 
È noto ad oggi come nessun essere umano possa preservare la propria condizione di salute o ambire a riconquistarla, se de-sincronizzato dai ritmi universali mediati dall'epifisi, quale fondamentale struttura immuno-stimolatoria la cui asportazione ha dimostrato di indurre un grave stato immunosoppressivo (6).

Diviene perciò possibile formulare la seguente affermazione: "Non esiste PNEI laddove sia escluso lo studio della ghiandola pineale".

In materia di epifisi, il tempo presente caratterizzato da importanti errori scientifico-intepretativi, dall'eccessiva valorizzazione del secondario e in particolare dall'omissione di ciò che è oggettivo, giunge non oltre la descrizione della melatonina ( $\mathrm{N}$-acetil-5-metossitriptamina), una soltanto tra gli infiniti composti pinealici bioattivi, quale ipno-favorente o antiossidante-anti-aging.

Ciò che in primis dovrebbe invece indurre a profonda meditazione, in seguito a un'attenta analisi della letteratura disponibile da decenni, è il ruolo anticancro della melatonina stessa, dimostrato dalla PNEI prima in condizioni sperimentali (7-11) e confermato successivamente in ambito clinico dai lavori del gruppo dell'Ospedale San Gerardo di Monza (12).

La melatonina presenta chimicamente uno scheletro indolico, caratteristica strutturale di un'altra molecola pinealica, la 5-metossitriptamina, la quale ha a sua volta dimostrato di esercitare in vitro un'attività anti-tumorale persino superiore a quella della stessa melatonina (13).

Gli indoli pinealici noti tra i quali figurano l' acido-5-metossi-indolacetico, il 5-metossi-triptofolo, la 5-metossi-triptamina e l' $\mathrm{N}$-acetil-5-metossi-triptamina o melatonina, secreti rispettivamente attorno alle ore di alba, mezzodì, tramonto e buio, mimano il rapporto corpo-coscienza che muta durante le ventiquattro ore, conservando peraltro una struttura, quella indolica, propria di infiniti alcaloidi dalle proprietà psichedeliche tra cui il DMT (dimetil-triptamina) contenuto nella Psycotriaviridis, la psilocina e la psilocibina isolabili dalle Psylocibeazurescens (Teonanacatl) ePsylocibecubensis, ritrovabili nel regno vegetale, labufotenina del Bufo alvariusriscontrabile nel regno animale e il più noto LSD derivato dall'universo della chimica di sintesi.

È ormai evidenza esistenziale confermata dalle recenti scoperte della PNEI in campo oncologico [14] che qualsivoglia condizione meditativo-spirituale, l'eccitazione sessuale e l'amplificazione del piacere [15] possano giocare un ruolo anticancro attraverso una doppia azione: da una parte attraverso l'inibizione diretta della proliferazione cellulare maligna, dall'altra mediante lo stimolo della naturale resistenza immunobiologica contro lo sviluppo neoplastico.

Ciò è naturalmente valevole per composti chimici aventi la proprietà di espandere lo stato di coscienza tra cui cannabinoidi [16], indoli pinealici [17] e beta-carboline [18], anche queste ultime prodotte dall'epifisi. Esse svolgono un ruolo chiave nei processi di autocoscienza e nella neurochimica della vita spirituale, trovando autorevoli analoghi strutturali fitochimici in composti con attività psicotropa come armina e armalina [19], beta-carboline isolabili da una liana del genere Banisteriopsiscaapi, originaria del Sud America.

La funzione antitumorale svolta dalle molecole pinealiche è associabile a meccanismi differenti ovvero un'azione citotostatica-citotossica diretta, un effetto stimolante sull'immunità anticancro mediata dai linfociti $\mathrm{T}$ e un'attività anti-angiogenetica, ossia di contrasto verso le neo-formazioni vascolari indispensabili per il nutrimento della neoplasia [19]. La ghiandola pineale si rivela quindi come primo organo anticancro del corpo umano, la PNEI come scienza che ne studia il ruolo di direzione orchestrale neuro-endocrino-immunitaria. 
Dal riconoscimento della funzione epifisaria è possibile giungere ad un'evoluzione successiva concependo l'attività pinealica come inseparabile da quella del sistema interneuronale cannabinoide. I cannabinoidi endogeni, i più noti tra i quali sono rappresentati da anandamide o arachidonil-etanolammide e 2-arachidonil-glicerolo, hanno pure dimostrato di esercitare un'azione antineoplastica su diverse linee cellulari, mediante un'attività diretta antiproliferativa e anti-angiogenetica, così come attraverso l'inibizione della risposta infiammatoria cronica mediata dai macrofagi, nota per agevolare la disseminazione metastatica [20].

\section{“Risulta quindi comprensibile il rivoluzionario principio della PNEI che afferma come tutto ciò che espanda la coscienza sia al contempo anticancro.”}

Parimenti, azioni antitumorali sono state osservate per l'ormone natriuretico atriale o ANP [21] secreto dal cuore in quanto ghiandola endocrina e per l'ossitocina secreta dalla neuroipofisi [22-23]], ormoni entrambi presentanti simultaneamente anche una marcata azione neuro-psichica.

L'ultima rivelazione chimica in ordine cronologico che ha saputo contribuire al progresso delle conoscenze in ambito di PNEI è senza dubbio la ri-scoperta dell'attività del prodotto enzimatico della ACE2 (Angiotensin-Converting-Enzyme2), l'angiotensina 1-7, peptide dotato di attività antitumorali e antiinfiammatorie [24].

La ricerca moderna in materia dimostra in sintesi come le attività delle singole strutture deputate alla fisiologica rigenerazione siano da concepirsi non come isolate bensì come strettamente interdipendenti nel generare una rete neuro-endocrina presieduta dalla ghiandola pineale, finalizzata in ultimo alla fine regolazione del sistema immunitario, attraverso la produzione e conseguente immissione nel sangue dei secreti caratteristici.

È necessario però compiere un ultimo passaggio fondamentale ai fini della comprensione del un corpo umano nella sua totalità, sia esso contemplato nella fede quale immagine di Dio o considerato in scienza e coscienza come "intermedio di reazione" di un'evoluzione tuttora in fieri, vale a dire quello dello studio delle citochine, ovvero glicoproteine mediante le quali, le cellule immunitarie attivate comunicano reciprocamente a distanza, ma non solo. La PNEI ha infatti dimostrato come le citochine non influenzino esclusivamente il sistema immunitario bensì la totalità dei sistemi biologici tra cui il metabolismo, il sistema cardiovascolare e la funzione stessa dell'intero sistema neuro-endocrino.

La palese complessità del network citochinico risiede nel fatto che l'interazione sussistente tra le singole interleuchine, alcune delle quali presenti in differenti isoforme e identificate in un numero complessivo che oltrepassa ad oggi il quaranta [24], è di tipo a feedback positivo, ossia caratterizzato da stimolazione reciproca, laddove il sistema endocrino è notoriamente riconosciuto dall'endocrinologia classica come fondato in larga parte su circuiti a feedback negativo.

Tutte le funzioni biologiche si presentano quindi sotto il controllo di due fondamentali sistemi regolatori che comprendono sistema endocrino e network citochinico.

Alla luce di una tale consapevolezza i fondamenti fisiologici della PNEI possono essere riassunti da tre evidenze strutturanti:

1. le cellule immunitarie, linfociti e macrofagi presentano recettori per ormoni e neuromediatori, rivelandosi come sottoposti a un controllo centrale di tipo neuro-endocrino, e a loro volta, attraverso la produzione di citochine, sono in grado non solo di agire sulle restanti cellule immunitarie, bensì anche da una parte sul sistema psico-neuro-endocrino, dall'altra sullo stesso metabolismo; 
2. le cellule endocrine e nervose sono provviste di recettori per le citochine e producono composti neuro-attivi e ormoni, in grado di presentare non solo naturali funzioni neuroendocrine, ma di incidere contemporaneamente sulla funzionalità delle cellule immunitarie con effetti stimolatori o inibitori;

3. le cellule immunitarie possono a loro volta produrre ormoni e neuro-mediatori, mentre specularmente le cellule nervose ed endocrine producono citochine.

Le cellule immunitarie stesse, in particolare i linfociti, possono essere dunque considerate come sensibilissime micro-ghiandole endocrine circolanti all'interno del corpo biologico, il quale si rivela governato essenzialmente da dinamiche simil-endocrinologiche.

L'esistenza e l'indagine di un condizionamento della vita psico-spirituale sullo stato di salute del corpo presuppongono quindi una solida conoscenza dei meccanismi neuro-immuno-chimici attraverso cui vissuti emozionali e spirituali vengono a condizionare inevitabilmente la biologia del corpo fisico.

Infine, il terzo e ultimo livello di comprensione della PNEI ne prevede la piena attuazione cosciente quale PNEI clinica, ovvero la semplice applicazione alla clinica delle conoscenze della PNEI, preceduta da una diagnostica di laboratorio nuova che indaghi da un lato la funzionalità dei principali organi deputati al controllo neuro-endocrino, dall'altro l'attività citochinica sistemica quale specchio elettivo della funzione immunitaria, con un unico e solo intento sintetico nei confronti del malato: ristabilire la chimica neuro-immuno-endocrina dello stato di salute, ottemperando di fatto all'antico principio alchemico del "Natura Naturamadiuvat", confermato dallasapienza avestico-islamica e divenuto definitivamente scienza con le scoperte della PNEI contemporanea, mediante l'attestazione di come in natura sia stato posto il rimedio ad ogni umana sofferenza.

È possibile a tal proposito il ricorso terapeutico da una parte a molecole, peptidi o proteine endogene del corpo umano, dall'altra a molecole o fitocomplessi provenienti dal regno vegetale, la cui attività acquista oggi nuovo valore in seguito allo studio dell'effetto finale sul bilanciamento dell'attività citochinica, indispensabile a decretarne la definitiva valenza curativa.

Dal punto di vista antropologico poi, il ristabilire l'equilibrio chimico neuro-endocrino dello stato di salute, principio fondativo riconosciuto dalla PNEI clinica come bene primario per il malato, è formulato a differenza di qualsivoglia approccio medico convenzionale o integrativo, sulla base di un modello originale, ovvero su di una concezione precisa di Essere Umano inteso come Trinità di Corpo, Anima o Psiche e Spirito, intendendo per Corpo sia la chimica metabolica, sia quella psiconeuro-endocrino-immunomodulante, per Anima o Psiche l'oceano di pulsioni che secondo il pensiero Freudiano hanno nella sessualità la matrice essenziale, e per Spirito, l'Amore, in quanto stato supremo della Coscienza, evangelicamente concepito come la percezione del prossimo da parte dell' uomo quale un altro sé stesso.

Come intuito dal profeta Zarathustra, nello stato definitivo di Santità ovvero di Amore, unito come dimostrato oggi dalla PNEI alla perfetta sincronia con le leggi universali trasdotte in biologia umana dall'epifisi, la Salute è condizione neuro-endocrino-immunitaria perfetta. 
Alla luce dell' odierno sapere in ambito non solo scientifico, la PNEI clinica si rivela in definitiva non solo come disciplina scientifica integrativa, bensì per meglio dire come medicina rigenerativa fondata sulla perfetta conoscenza biochimica delle naturali capacità auto-rigenerative del corpo umano, espresse dai medesimi organi predisposti sia alla percezione del Piacere che all'espansione della Coscienza, e mediate in ultimo da una chimica biologica non più qualificabile come psicotropa, bensì determinabile e ridefinibile come pneumotropa, in quanto "precipitato pneumo-chimico" attraverso il quale lo Spirito ovvero l'Amore si esprime e si auto-amplifica infinitamente.

Paolo Lissoni, AlejandraMonzon, Andrea Sassola

\section{Bibliografia}

1) Albert Hoffman, $\boldsymbol{L S D}$ il mio bambino difficile; Urra- IDEE editoriali FELTRINELLI S.r.1;

2) Alexander \& Ann Shulgin PIHKAL;

3) Alexander \& Ann Shulgin TIHKAL;

4) Wisneski LA, Psychoneuroimmunology: from biochemistry to energy medicine.

SubtEnergMed 11: 23-40, 2019;

5) Breziski A. Melatonin in humans. N Engl J Med 336; 186-195, 1997;

6) Jankovic BD, Isakovic K, Petrovic C. Effect of pinealectomy on immune reactions in rat. Immunology 18: 1-3, 1970;

7) Regelson W, Pierpaoli W. Melatonin: a rediscovered antitumor hormone? Cancer Invest 5: 379-385, 1987;

8) Bartsch C, Bartsch H. Melatonin in cancer patients and in tumor-bearing animals. Adv Exp Med Biol 467: 247-264, 1999;

9) Maestroni GJM. The immunoneuroendocrine role of melatonin. J Pineal Res 14: 1-10, 1993;

10) ReiterRJ. Mechanisms of cancer inhibition by melatonin. JH Pineal Res 37: 213-214, 2004;

11) Hill SM, Frasch T, Xiang S, Duplessis T, Mao L. Molecular mechanisms of melatonin anticancer effects. Integr Cancer Ther 8: 337-346, 2009. Blask;

12) Lissoni P, Rovelli F, Brivio F, Messina G, Lissoni A, Pensato S, Di Fede G. Five-year survival with high-dose melatonin and other antitumor pineal hormones in advanced cancer patients eligible for the only palliative therapy. Res J Oncol 2: 1-7, 2018;

13) Sze SF, Ng TB, Liu WK. Antiproliferative effect of pineal indoles on cultured tumor cell lines. J Pineal Res 14: 267-273, 1993.

14) Antony MH. Psychoneuroimmunology of cancer. Brain Behav Immunol.2003; 17:84-91;

15) Riley V. Psyconeuroendocrine influences on immunocompetence and neoplasia. Science. 1981; 212:1100-1109;

16) Grotenhermen F. Pharmacology of cannabinoids. Neuroendocrinol Lett.2004; 25:14-23;

17) Maestroni GJM. The immunoendocrine role of melatonin. J Pineal Res. 1993; 14:1-10;

18) Ishida J. Wang HK, Bastow KF, Hu CQ, Lee KH. Antitumor agents 201.Citotoxicity of harmine and beta-carboline analogs. Bioorg Med Chem Lett 1999; 9:3319-3324;

19) Conti A, Maestroni GJM (1995) The clinical immunotherapeutic role of melatonin. J Pineal Res 19(3);103-110; 
20) Grotenhermen F (2004) Pharmacology of cannabinoid Neuro Endocrinol Lett 25(1-2): 1423 ;

21) Kong X, Wang X, Xu W, Behera S, Hellermann G, Kumar A, et al. (2008) Natriuretic peptide receptor A as novel anticancer target. Cancer Res 68(1): 249-256;

22) Murrel TG(1995) The potential for oxytocin (OT) to prevent breast cancer: a hypothesis. Breast Cancer Res Treat.35(2):225-229.

23) Cassoni P, Marrocco T, Deaglio S, Sapino A, Bussolati G (2001) Biological relevance of oxytocin and oxytocin receptors in cancer cells and primary tumors. Ann Oncol 12(Suppl 2):S37-39;

Psico - Neuro - Endocrino - Immuno- Somatologia (Dott. Lorenzo Paride Capello)

L'Olismologia $^{\circledR}$ è un modello clinico innovativo che introduce nell'attuazione del paradigma PNEI - in essa interamente applicato - anche la componente "Struttura" (PNEI-S); ciò permette di esaminare finalmente ogni singola Persona nella sua totalità e di definire per ciascun Paziente il piano terapeutico più completo e mirato: tutto ciò in un atto medico unitario e simultaneo (N.B: non unico e definitivo).

Da molto tempo si parla di P.N.E.I. e infiniti studi hanno accertato che tutto informa tutto, che le cellule di ciascun sistema - nervoso, neuro-endocrino e immunitario - sono dotate di recettori e mediatori chimici che permettono loro di comunicare, di informarsi e di interagire. Il concetto che lo squilibrio di qualunque turba dell'organismo riverbera sull'intero Essere ci richiama all'unità di quel tutto che da tempo abbiamo spezzettato, perdendo la capacità di sintesi e la visione d'insieme dell'Essere umano.

Gli Specialisti- solidamente competenti ma nel contempo confusi e paralizzati dalla crescente complessità delle loro conoscenze- guardano sempre meglio in un ambito sempre più piccolo e continuano ancor oggi (senza tener conto dell'interdisciplinarietà della P.N.E.I.) a esaminare soltanto alcune parti e funzioni corporee, trascurando sovente il più vasto contesto in cui le patologie si generano.

La comprensione della grandiosa complessità del nostro essere e del nostro divenire induce - ora più che mai - a ricordare l'antico termine olismo che anticipava la visione olistica attuale della Medicina energetica, funzionale, vibrazionale.

In Olismologia ${ }^{\circledR}$ parlo di PNEI-S, ovvero di psico-neuro-endocrino-immuno-SOMATO-logia, perché TUTTO il CORPO nel suo insieme è l'attore unico e il protagonista competente del nostro RIequilibrio vitale GLOBALE. 


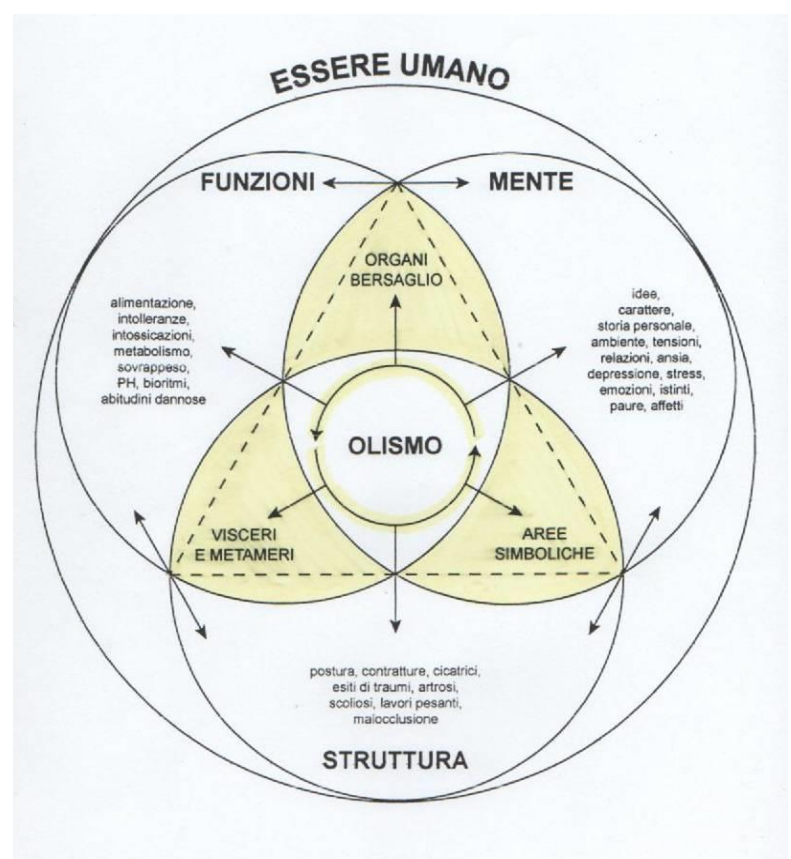

N.B. Quando scrivo la parola Corpo con la lettera maiuscola esprimo il concetto che il nostro organismo - in virtù della sua sapienza innata - deve essere considerato il soggetto protagonista dell'indagine, e non un oggetto passivo.

Non è più sufficiente parlare di P.N.E.I.; occorre chiudere il cerchio del concetto di globalità dando finalmente dignità anche alla componente Strutturale.

L'aggiunta della S di Soma all'acronimo, sottolinea la fondamentale importanza che la componente meccanicaassume nella genesi e nella spiegazione dei fenomeni psico(P.N.) -bio(E.I.) -somatici(S.), per le evidenti conseguenze che l'alterazione dello stato di tensione o di rilassamento del sistema muscolo-fasciale produce sull'equilibrio psico-fisico globale.

Nella mia attività professionale quotidiana ho ampiamente accertato che la Struttura dell'Essere Umano - alla quale è stato sempre assegnato il ruolo ininfluente di mero "apparato locomotore e di sostegno" - possiede invece, al pari dei visceri, tutte le caratteristiche di un organo-bersaglio e comunica anch'essa con tutto il resto dell'organismo. Anch'essa risente degli stimoli emozionali e determina rilevanti conseguenze sul piano Funzionale e Mentale.

Infatti - sul piano Strutturale, della meccanica e della dinamica corporea - ogni vissuto si accompagna con un atteggiamento fisico (mimica posturale) e/o con qualche sintomo sgradevole eloquente, proprio come a ogni emozione corrisponde un caratteristico atteggiamento mimico del volto.

Non soltanto la Mente - dunque - genera disturbi funzionali a carico degli organi-bersaglio, ma anche la muscolatura, poiché le emozioni si traducono in contratture, tensioni e rigidità che compromettono l'efficienza delle unità neuro-vascolari.

\section{IL DISTURBO FUNZIONALE}

Tutti abbiamo provato quale effetto provoca lo stare seduti scomodi o in una posizione scorretta: all'atto di alzarsi non si riesce ad appoggiare in terra il piede perché la gamba formicola e costringe 
a zoppicare. Nel giro di 2 o 3 minuti la parestesia scompare e si riprende a camminare normalmente. È palesemente un disturbo funzionale temporaneo e transitorio, dipendente dallo schiacciamento di un nervo (l'unità neuro-vascolare, per l'appunto).

Con la stessa modalità insorgono numerosi altri disturbi che "vanno e vengono".

Una patologia quando c'è, c'è; non c'è ora sì/ora no.

Pertanto prima di trattare i sintomi remittenti come fenomeni patologici stabili, è bene ricordare che buona parte della patologia è in realtà fisiologia dimenticata, negata, contrastata, repressa che può essere ripristinata.

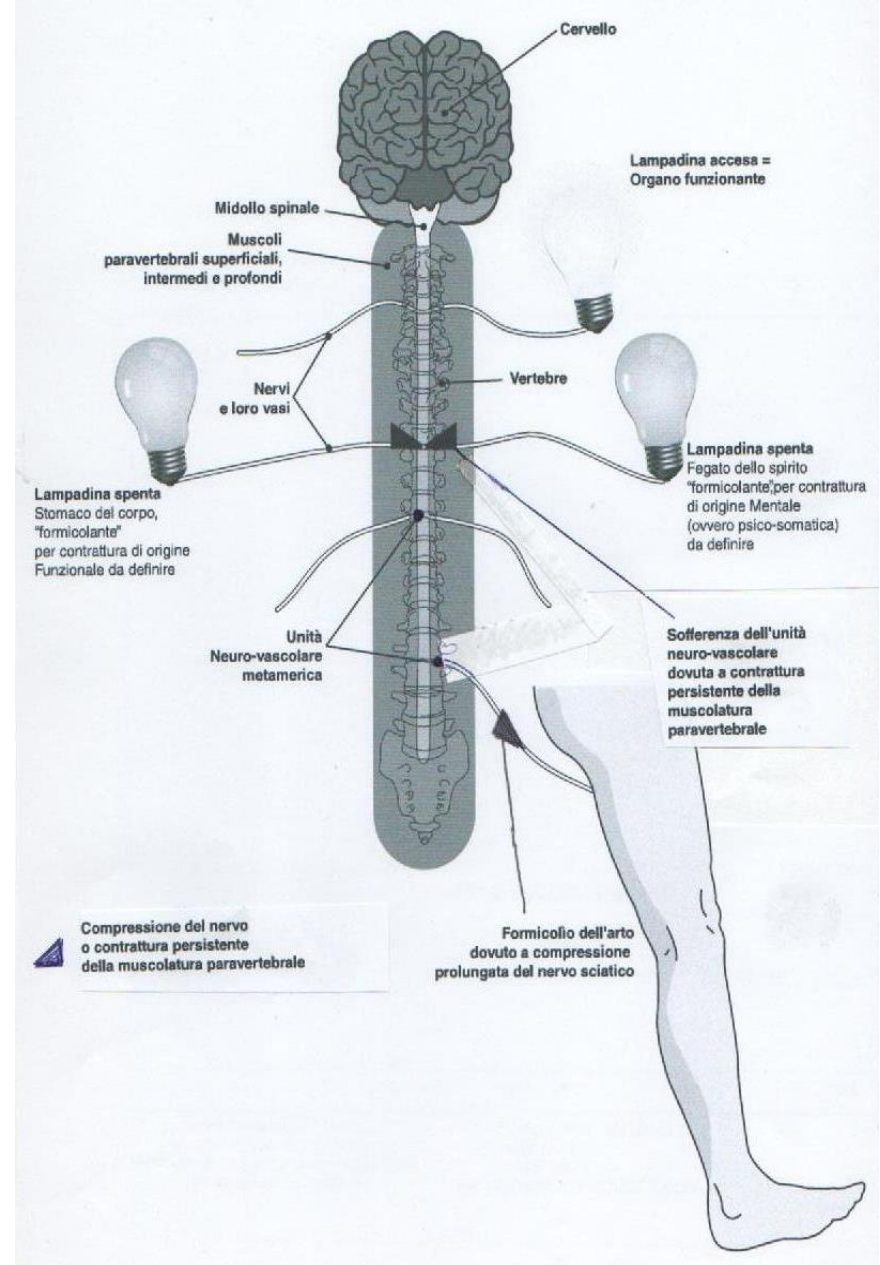

\section{La MANIPOLAZIONE CLINICA a livello metamerico cura le disfunzioni degli organi e cura i problemi emotivi e/o psico-umorali attraverso le Aree Simboliche.}

Il Corpo è la rappresentazione materiale della Mente.

Le informazioni che provengono dal cervello vengono tradotte meccanicamente $\mathrm{e}$ inconsapevolmente "registrate" - in direzione psico-somatica - nella Matrice extracellulare e nel tessuto fasciale del nostro corpo.

Definisco pertanto Mimica Posturale il risultato degli innumerevoli e impercettibili adeguamenti strutturali muscolo-fasciali che sul piano corporeo si associano automaticamente a ogni contenuto emotivo e determinano la formazione di zone di rigidità intrise di emozioni dolorose trattenute, inespresse, controllate, di esperienze di sofferenza non risolte, rimosse o negate. 
Ho denominato tali zone AREE CORPOREE SIMBOLICHE in quanto costituiscono una valida chiave di lettura per decifrare il linguaggio del corpo e per risalire ai contenuti emotivi che disturbano e/o affliggono la Persona.

La Manipolazione Clinica, agendo su queste aree di rigidità, produce un effetto di sbobinamento ovvero di riconversione in direzione somato-psichica - delle registrazioni corporee in emozioni ancora dolorose, vive, presenti e brucianti.

I contenuti delle Aree - sopiti, dimenticati, ignorati o trascurati - riaffiorano in modo incontenibile e con illuminante evidenza sul piano della coscienza, per cui il Paziente riesce ad affrontarli consapevolmente, comprendendo in modo estremamente chiaro la relazione tra i disturbi sofferti e le loro reali cause.

\section{MAPPADELLEAREE CORPOREE SIMBOLICHE}
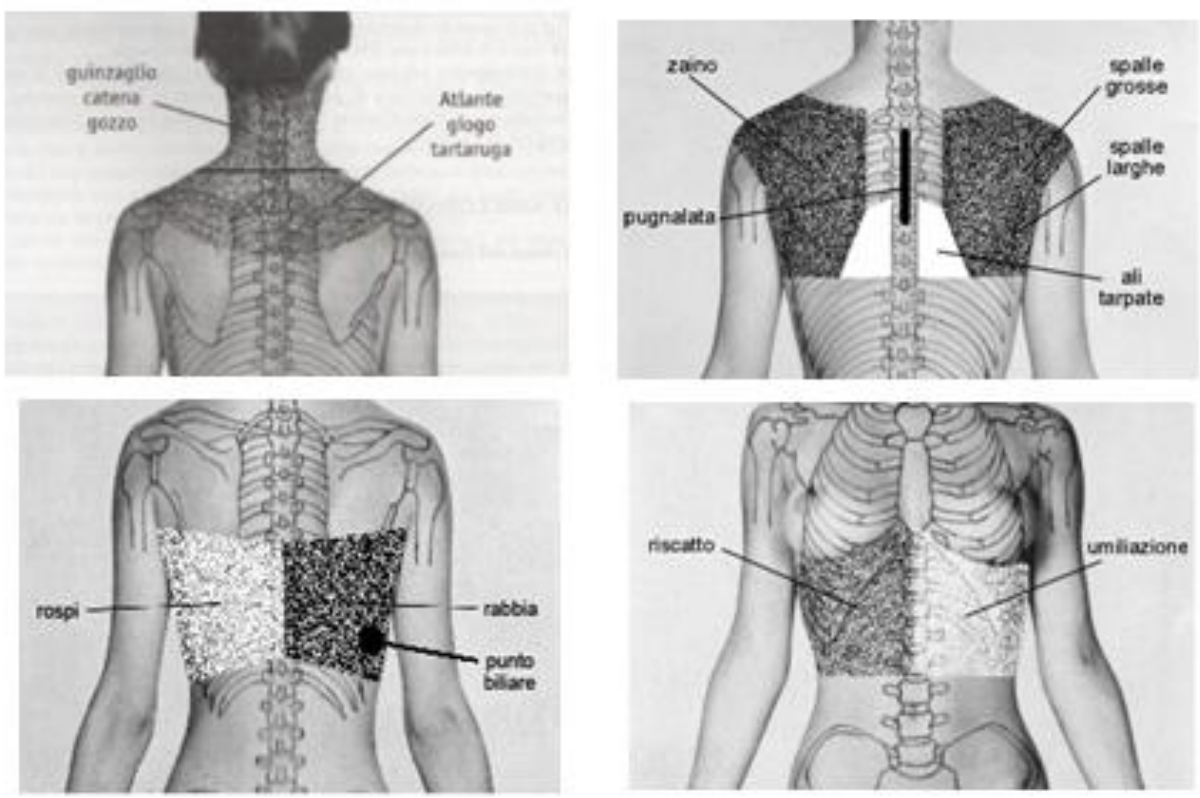

Per fare un esempio, l'Area simbolica della mantellina (la prima figura nel primo gruppo di 4 figure) indica condizioni di sopportazione volontaria, intenzionale. Offre vari spunti di lettura: la presenza di costrizioni esterne (guinzaglio), la mancanza di libertà (catena), l'incapacità o la paura di esprimersi (tener le cose sul gozzo), gravi preoccupazioni (Atlante, il mitico gigante che portava l'intero mondo sulle spalle, l'assunzione di troppi impegni o responsabilità (giogo), la codardia o la tendenza a sfuggire dalla realtà (tartaruga).

Nella parte inferiore del dorso, (visibile nella prima figura del secondo gruppo) si trovano le Aree simboliche della sella e della soma, quelle su cui si caricano i pesi agli animali - la sella al cavallo e la soma al somaro - i quali sono costretti loro malgrado a portarli. Rappresentano la sopportazione passiva, involontaria, l'insoddisfazione, l'insofferenza, la voglia di sgroppare situazioni o rapporti con persone che sono causa di stress prolungato e producono tensione; vi si trova la rassegnazione, la paura di ribellarsi, il servilismo, la vigliaccheria subita in nome del quieto vivere. Possono diventare l'approfondimento dei problemi legati alle aree gastrica ed epatica.

Al limite superiore dell'Area della soma sinistra c'è l'Area dell'aborto. Nelle donne è rilevabile dolorosissima - allorché siano state costrette a decidere o a subire l'interruzione di una gravidanza; più in generale, in entrambi i sessi, indica importanti progetti falliti, malamente abortiti. 


\section{MAPPA DELLE AREE CORPOREE SIMBOLICHE}
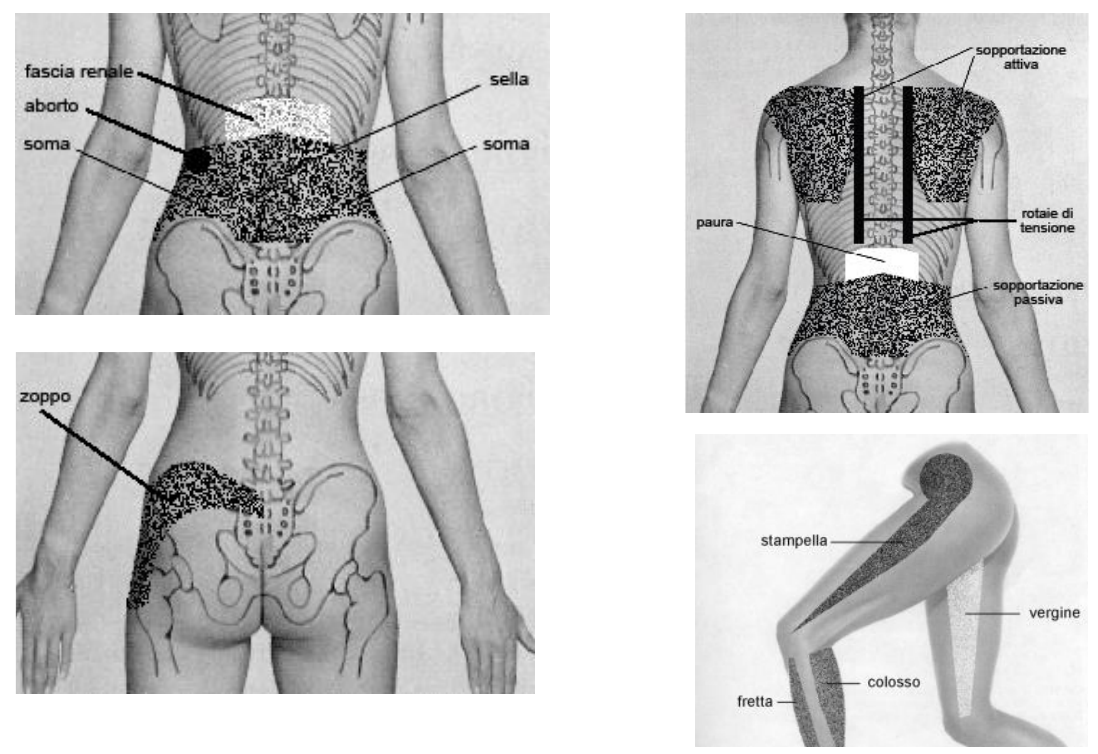

Desidero esporre il mio concetto di "Struttura" anche sul piano energetico.

Tutto ciò che si trova intorno a noi, fra noi, dentro e fuori di noi, ogni più piccola porzione di materia è composta da un numero incalcolabile di atomi e molecole, di elettroni, protoni e neutroni che vibrano nello spazio vuoto.

Solo un miliardesimo della realtà è costituito dalla massa, tutto il resto è costituito da biofotoni, cioè da energia; anche l'Essere Umano - al pari dell'intero Universo - è fatto di energia, ovvero di onde elettromagnetiche in vibrazione.

I Medici con l'elettro-cardiografo, l'elettro-encefalografo e l'elettro-miografo registrano l'attività elettrica delle cellule cardiache, nervose e muscolari. Anche la Risonanza Magnetica Nucleare invia stimoli elettromagnetici al campo elettromagnetico corporeo, affinché le cellule risuonino e rimandino quei segnali che consentono di disegnare la mappa degli organi interni.

La Fisica Quantistica sta unificando la dimensione mistica, la Filosofia e la Scienza; applicata alla materia vivente, inoltre, ha già provocato forti cambiamenti nel campo della Chimica, della Biologia e della Psicologia, e ora consente di comprendere molti contributi che provengono dalle Medicine Complementari, da tempo aperte a questa visione.

La Medicina istituzionale, invece, continua a valutare le alterazioni chimiche e meccaniche per impostare terapie farmacologiche e chirurgiche, sottovalutando il fatto che siamo, abbiamo e produciamo un campo elettromagnetico!

Eppure è così evidente cosa succede quando l'energia se ne va dall'essere vivente: resta un cadavere!

L'Olismologia $^{\circledR}$, Disciplina della Sintesi, non è solamente una forma mentis e un modus operandi innovativo, ma anche un concreto approccio clinico diagnostico-terapeutico che indaga il Network 
Psico-Fisico del Paziente, decodifica sul piano vibrazionale il linguaggio criptico dei segnali del suo Corpo e permette al Medico di interpretare e riequilibrare la "fisio-logica" coerente e illuminante con cui esso fa tutto, con l'innata sapienza della Vis Medicatrix Naturae.

Il Network del Paziente è costituito dal complesso intreccio onnipresente di vie nervose e linfatiche periferiche, nella quale il liquor cerebrospinale (che accompagna le strutture del sistema nervoso fino ai filuzzi più lontani) e la linfa (che si genera nel tessuto connettivale) si mescolino (come avviene quando un apparecchio elettrico cade nell'acqua) generando l'Hardware del campo elettromagnetico corporeo. Allo stesso modo, le bioinformazioni (biofotoni) si trasmettono nella Matrice Extracellulare in ogni distretto corporeo.

Questa immagine rende l'idea di come il Software Funzionale e Mentale sia in grado di mantenersi in contatto istantaneo e dinamico con l'Hardware, nonché di rispondere agli stimoli con velocità fulminea e in modo coordinato, ben diversamente da quanto avverrebbe se la trasmissione dei segnali fosse basata su lente reazioni biochimiche. L'istantaneità della biochimica appresa sui banchi di scuola acquista senso solo quando la si comprende coordinata energeticamente in modo coerente.

\section{Il campo elettromagnetico dà forma alla materia e la governa, così come una calamita dà forma e governa un mucchietto di limatura di ferro.}

Per chiarire ulteriormente i concetti Olismologici dell'Hardware e del Software Corporei e approfondire quindi la PNEI-S,mi piace fornire ulteriori elementi.

\section{Hardware Strutturale}

Un computer è fatto di plastica, cristalli liquidi e materiale elettrico vario, inerti. Quando è guasto lo si getta via. Similmente noi siamo fatti di acqua, carne, ossa, connettivo e visceri, sostanze materiali inerti, da sotterrare dopo la morte come un vuoto a rendere. Il Corpo rappresenta "la torre" del computer, con l'hard-disk e tutti i suoi componenti operativi. È per l'appunto il nostro "Hardware Strutturale". In esso risiede una sapienza plurimillenaria antecedente a qualunque teoria, libro o conoscenza medica: è la fonte primaria della Vis Medicatrix Naturæ. Su questo piano occorre procedere materialmente, con cacciavite, pinza e saldatore, ovvero con le mani.

\section{Software Funzionale}

Quando il computer viene collegato all'impianto elettrico è in grado di funzionare, e quando l'accendiamo i suoi circuiti si attivano. Allo stesso modo, quando l'energia vitale anima il nostro Corpo noi viviamo e i nostri visceri svolgono le funzioni organiche.

Tutto ciò che porta all'Hardware Strutturale informazioni e regolazione, come i vari accessori esterni USB, rappresenta il nostro "Software Funzionale", la componente energetica, sulla quale agiscono le cure vibrazionali, mirate su ogni singolo Paziente. Le cure sono i nuovi programmi che si installano per correggere quelli in uso non più efficienti, che manifestano (con sintomi organici) palesi disfunzioni operative e/o errori di elaborazione.

\section{Software Mentale}

Quando apriamo un sito per navigarlo, il computer ce lo mostra. Così pure noi, con la Mente, formuliamo i nostri pensieri. Mentre il computer non sa cosa trasmette, perché è passivo, indifferente, 
noi invece possiamo scegliere con consapevolezza - come spettatori esterni - quale sito navigare, e possiamo cambiarlo, se non vale. È il nostro "Software Mentale", sul quale agiscono le cure vibrazionali, mirate su ogni singolo Paziente.

In Olismologia ${ }^{\circledR}$ le terapie vibrazionali vengono identificate con l'Estimologia Clinica.

Affinché tutte le operazioni del Software Funzionale e Mentale possano essere eseguite compiutamente, è necessario che l'Hardware Strutturale sia preliminarmente attivato e reso efficiente.

Così come è impossibile pretendere che un PC spento o in standby elabori i vari software che gli vengono inseriti (USB), allo stesso modo è difficile per un Corpo torpido e inefficiente elaborare le sostanze curative che gli vengono somministrate.

L'Essere Umano è un ecosistema multifunzionale coerente, di natura vibrazionale in cui tutto STRUTTURA, FUNZIONI e MENTE- è collegato (PNEI-S) e totipotente, grazie alla Vis MedicatrixNaturæ.

La salute è la condizione di equilibrio in cui ogni Persona vive quando tutte le reazioni chimiche e le funzioni organiche si svolgono in modo coerente.

Quando il Corpo si trova in condizioni di squilibrio (stato di malattia) esprime il proprio disagio con un linguaggio criptico, fatto di segnali - sintomi fisici o psichici - che devono essere riconosciuti e interpretati correttamente, nonché risolti con un linguaggio vibrazionale e con soluzioni compatibili con la sua Fisiologia.

CURARE significa ristabilire la piena coerenza tra le funzioni corporee, fisiche e psicosomatizzate.

\section{Bibliografia}

Web: $\underline{\text { www.olismologia.it }}$

Web: https://www.aspicpsicologia.org/ricerca-scientifica/lintegrazione-somatopsichica.html

Gold J. R. (2000), Le basi dell'integrazione, Sovera, Roma.

Piroli C. (2001), Enciclopedia medica - Nuovi orientamenti e scoperte, II corpo si risveglia: l'Integrazione Posturale dinamica. Ed. IPS srl, Roma.

Piroli C., Montanari C., lannazzo A. (2010), II Corpo Integrato. Teorie e Tecniche Psicocorporee nella Psicoterapia e nel Counselling, Ed. Scientifiche ASPIC, Roma.

Reich W., (1994), L'analisi del carattere, SugarCo, Milano.

Rogers C. (1970) La terapia centrata sul cliente, Martinelli, Firenze. 


\section{La visione Sistemica attraverso la Consapevolezza (Fabbroni Roberto)}

Per quanto riguarda la PNEI-S vi sono altre tipologie di approcci che tendono a lavorare sul corpo per rimodulare lo stato psicologico e oltre all'analisi Bioenergetica che abbiamo ben argomentato nella parte dedicata alla Psicosomatica che fa parte, in linea generale, di quel filone che viene chiamato Psicoterapia a Mediazione Corporea, troviamo la Tecnica Alexander che approfondiremo a breve.

Infatti, al contrario della maggior parte delle psicoterapie, che curano i mali dello spirito attraverso il linguaggio, le psicoterapie corporee lo fanno passando per il corpo attraverso svariate tecniche come il massaggio o il rilassamento.

Le diverse terapie a mediazione corporea possono integrare svariati approcci sia come vere e proprie psicoterapia (Analisi Bioenergetica e Tecnica Alexander), sia attraverso l'integrazione di alcune discipline naturali.

Ad esempio tra queste possiamo trovare i metodi di rilassamento, messi in pratica dagli osteopati che cercano di ridurre le tensione psicologica attraverso l'apprendimento del rilassamento muscolare. Il training autogeno si concentra sull'apprendimento del rilassamento muscolare e della concentrazione sulle sensazioni. Si fanno provare al paziente delle sensazioni perdute, affiché possa recuperare il controllo sul proprio corpo.

I massaggi permettono di distendere il corpo, di eliminare le tensioni legate spesso allo stress, soprattutto nella schiena e nel collo. Il sollievo procurato provoca una sensazione di benessere. È un buon modo per accompagnare un paziente nel suo percorso personale. Tra i vari metodi di trattamento, citiamo lo shiatsu, che opera attraverso pressioni su alcune zone del corpo.

La sofrologia modifica lo stato di coscienza, ma senza raggiungere l'ipnosi. Il paziente viene spinto all'isolamento sensoriale per favorire la sua immaginazione, il che contribuisce al suo equilibrio. La riflessologia parte dal principio che vuole che tutte le parti del nostro corpo si proiettino sul piede in punti riflessi. Massaggiando questi punti si liberano le tensioni dell'organismo.

L'intrecciarsi delle vicende del corpo da una parte e della psicoterapia dall'altra costituisce una storia che ha radici molto antiche e che, a ben guardarla, ha un andamento progressivo, un chiaro trend, verso nuovi interessanti sviluppi teorici e tecnici. La psicoterapia corporea è un insieme di metodologie, di tecniche, di ipotesi, che si caratterizzano non tanto per avere come loro oggetto il corpo, ma per uno studio approfondito delle relazioni tra mentale e corporeo, per una teoria di fondo che ipotizza l'unitarietà dello psiche-soma, superandone il tradizionale dualismo. Viene messa in evidenza la circolarità e la pariteticità delle correlazioni tra questi due aspetti della persona, al posto di una visione di tipo piramidale, di un corporeo sottomesso ad un mentale che "dall'alto" controlla l'intero funzionamento dell'organismo. La psicoterapia corporea è dunque una delle grandi aree teoriche che costituiscono oggi la psicoterapia. 
Intervenire direttamente sul corpo, infatti, in terapia ha messo in luce una serie di fenomeni e aspetti nuovi del funzionamento psicofisico che non erano stati osservati dagli altri approcci clinici. Si tratta di fenomeni forti e rilevanti, particolarmente significativi: esplosioni emozionali di grande intensità; posture, movimenti e toni di voce estremamente "regressivi"; ritorni di percezioni e ricordi arcaici; stati di coscienza diversi e profondi; riedizioni benigne di antichi sintomi; modificazioni evidenti delle funzioni fisiologiche interne (temperatura, frequenza del battito, sudorazione, soglie percettive, tono muscolare di base, peristalsi, processi ormonali); e così via. Tutto ciò ha messo sotto nuova luce le connessioni tra psichico e somatico, ponendo la necessità di inquadrare tali fenomeni in una nuova prospettiva, in nuovi principi, in nuove teorie. E questa sta producendo un vero e proprio terremoto nella psicoterapia poiché conduce via via ad una radicale ridefinizione dei concetti classici e alla formulazione di nuovi, in una risistemazione dell'intero campo teorico che non riguarda certamente il solo settore psicocorporeo ma tutta l'area della psicologia clinica e della psicologia più in generale. E nelle scienze dell'uomo la psicoterapia corporea potrà portare sempre più i suoi insostituibili contributi ricordando sempre che una psicoterapia è un delicatissimo processo complessivo nel quale sono indispensabili un'esperienza profonda di regressione, un poter essere accolti, presi e tenuti totalmente, un ripercorrere le tappe della propria vita per trasformarne gli esiti negativi attraverso la trasformazione del transfert, una "riparazione" attraverso la relazione con il terapeuta di antiche "lacerazioni" nel tessuto del Sé, un percorso e una crescita dal bisogno e la dipendenza al benessere e all'indipendenza.

Dunque anche la psicoterapia corporea non può ridursi assolutamente ad un insieme di tecniche, ad un'applicazione meccanica di esercizi, ma si svolge e realizza i suoi obbiettivi grazie alla relazione con il terapeuta, ad una teoria della relazione, che potremmo dire Energetico-Informazionale, ad un uso metodologicamente ed eticamente corretto di tale relazione, ad una modificazione graduale ma profonda delle caratteristiche di questo rapporto particolare, dall'inizio via via sino alla sua conclusione.

Completiamo la nostra analisi su un altro di questi approcci a mediazione corporea la Tecnica Alexander che è un altro approccio famoso a livello internazionaleche attraverso il corpo rimodula lo stato d'essere della persona in un processo di rieducazione che aiuta a diventare più consapevoli dell'equilibrio, della postura e del movimento nelle attività quotidiane utile come conferma di quanto fin qui visto. Tale metodo aiuta a rilasciare le tensioni muscolari superflue che, inconsciamente, sono divenute parte del modo abituale di muoversi. Rende più sensibili ed accurate le proprie percezioni e consente di coordinare e distribuire meglio le tensioni muscolari necessarie.

La Tecnica Alexander agisce, quindi, a livello psico-fisico; attraverso una serie programmata di esercizi e indicazioni si creano le migliori condizioni per lo svolgimento di qualsiasi azione motoria nella maniera più coordinata possibile con il minimo sforzo, la massima facilità e in modo consapevole mantenendo sempre uno stretto controllo per il corretto equilibrio posturale.

Essa consiste in vere e proprie sedute in cui si insegna al paziente a muovere la schiena (considerata l'elemento fondamentale del corpo) nella modalità corretta, secondo il principio per cui 'l'uso influisce sulla funzionalità". Ci sono, dunque, modalità di usare il proprio corpo che si rivelano migliori di certe altre. Tanto più ci si allontana dalla maniera ottimale tanto più le varie funzioni cominciano a soffrirne. Cioè potremmo dire che entriamo in uno stato di disequilibrio, di incoerenza. 
Spesso si è talmente abituati ad usare male il proprio corpo che tutto ciò risulta normale e "naturale"; non ci si rende conto che quotidianamente si tende a peggiorare il proprio quadro posturale e la funzionalità delle parti messe maggiormente sotto stress da un cattivo uso. Col tempo, secondo la Tecnica Alexander, le pressioni fisiche ed emotive che si accumulano si trasformano in tensioni croniche e "deformano" la struttura corporea e mentale. Ecco che la Tecnica Alexander riesce a fornire tutte quelle conoscenze necessarie per cambiare le proprie abitudini e organizzare in modo utile e vantaggioso la propria consapevolezza corporea.

In una seduta della Tecnica Alexander vengono combinate delle indicazioni verbali con delle sofisticate indicazioni propriocettive (derivanti dalle sensazioni tendinee, muscolari, posturali) trasmesse da leggerissime pressioni o "direzioni”" impartite dalle mani dell' insegnante.

La Tecnica Alexander incontrò fin dal principio il favore di numerosi medici e scienziati, tra cui Sir Charles Sherrington, padre della moderna neurofisiologia, che nel 1946 scrisse: Alexander ha reso un servizio all'argomento (la fisiologia della postura e del movimento) trattando insistentemente ogni atto come riguardante l'insieme psicofisico della persona. Fare un passo non è un affare di questa o quella gamba ma della totalità neurofisiologica del momento, non di meno della testa e del collo.

Nel 1937 un gruppo di 18 medici inglesi pubblicò sul British Medical Journal la richiesta che la Tecnica Alexander venisse inclusa nella formazione medica. Altri studiosi, tra cui il biologo americano George Coghill (1941) e l'antropologo Raymond Dart (1970) apprezzarono personalmente gli effetti della Tecnica Alexander e ne sostennero il carattere scientifico attraverso i loro scritti. Il professor NikolaasTimbergen, Premio Nobel per la medicina e la fisiologia nel 1973, dedicò buona parte del suo discorso onorifico alla Tecnica Alexander, raccomandandone l'adozione come "forma estremamente sofisticata di riabilitazione".

L'effetto terapeutico della Tecnica Alexander è tuttavia indiretto, secondario ai cambiamenti nell'uso di sé stessi. L'insegnante non cura i sintomi, bensì aiuta a rimuoverne le cause psicologiche che ne sono state la causa. I benefici vanno spesso al di là della risoluzione, ad esempio, del dolore e portano ad un senso di benessere inimmaginabile con la liberazione di endorfine.

Ora, alla luce di quanto appena visto quindi, sia attraverso la Psicosomatica, sia attraverso la PNEI e la PNEI-S, pare evidente che vi siano interconnessioni tra la Psiche, la componente Neurologica, il Sistema Endocrino, il Sistema Immunitario e il nostro Corpo/Soma. Queste intercorrelazioni vanno in ogni direzione. 


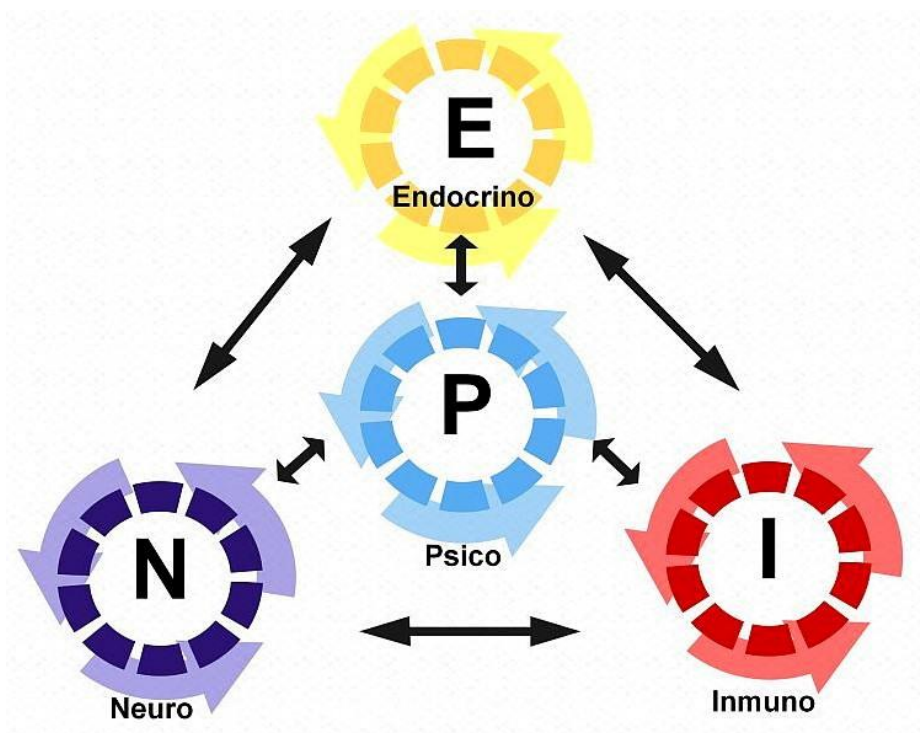

Se consideriamo l'uomo, quindi, in una visione sistemica, in un complesso espanso di ambiti materiali, immateriali ed energetici, la visione riduzionista a cui siamo abituati risulta in modo evidente,non più sufficiente né alla comprensione dei fenomeni, né alla determinazione dei processi. Questo comporta, necessariamente, un cambio del nostro punto di vista, una modifica delle metodologie, ed infine anche dei nostri comportamenti. In questa dinamica varia anche il paradigma al quale abbiamo affidato la nostra idea di salute e benessere.

La specializzazione medica, se da una parte ha sviluppato teorie che ci hanno permesso di scoprire alcuni principi fondamentali del funzionamento del sistema, dall'altro ci ha allontanati dalla visione totale dello stesso e quindi dalla visione sistemica, globale dello stato di salute dell'essere umano, proiettandoci in una visione riduzionistica dello stesso, correlata al lavoro sul sintomo e non sulla causa che in un siffatto contesto non può essere né vista né pensata.

La PNEI-S ci indica che in una visione Olismologican e ugualmente attraverso la Psicoterapia a Mediazione Corporea che tutto può essere visto in modo circolare e che a ritroso, attraverso un sistema di feedback, porta a valutare lo stato della Psiche attraverso il Soma che ne rappresenta, con la sua forma più o meno contratta, con la sua congestione energetica, la manifestazione più o meno salutare. In realtà la PNEI-S è compresa nella PNEI e l'aggiunta della S ha solo lo scopo di porre maggiore attenzione al "soma" per evitare che sia inteso solo come oggetto passivo di esternazione della parte psico-neuro-endocrina ma che esso ha una rilevanza paritetica con gli altri tre sistemi.

La moderna PNEI, porta in auge il ruolo della Ghiandola Pineale anche come mediatore Spirituale, intesa come espansione di coscienza e correlazione con tutto ciò che esiste $\mathrm{e}$ in modo particolare con le energie primordiali generatrici di questo spazio-tempo in cui ci muoviamo e respiriamo.

Parlare di Spirito in un contesto scientifico è appropriato e lo era anche in passato fino al '700 quando Cartesio con le sue scelte portò alla separazione tra Medicina-Scienza e Spirito-Religione.

Ricordo che il termine "psicologia" deriva dal greco psyché $(\psi v \chi \eta ́)=$ spirito, anima e da $\operatorname{logos}(\lambda o ́$ yos $)=$ discorso, studio, letteralmente ad indicare quindi lo studio dello spirito o dell'anima e rimase immutato fino al XVII secolo, quando assunse il significato di "scienza della mente" e negli ultimi cento anni è cambiato ulteriormente adeguandosi alle nuove prospettive e alla moderna metodologia. 
Ora è importante riprendere la visione unitario od olistica della salute umana recuperando la Consapevolezza della connessione esistente tra Psiche, Corpo, Mente e Spirito (o se preferite EnergiaInformata).

\section{Teoria del Campo di Consapevolezza Unificato - TCCU (Fabbroni Roberto)}

L'interazione tra Psiche, Corpo, Mente e Spirito quindi abbiamo visto, in questa lunga disamina che ci porta a manifestazioni fisiologiche, somatiche e che tale stato complessivo è spiegabile, come vedremo, attraverso la Teoria del Campo di Consapevolezza Unificato - TCCU.

LaTCCU indica nella Consapevolezza umana il mezzo per la gestione della salute umana, del suo "Ben-Essere", consentendo la spiegazione dei meccanismi che sottostanno alla Psicosomatica, PNEI e PNEI-S: meccanismi di tipo elettromagnetico e non solo.

Lo stato di Benessere, secondo la TCCU è lo Stato di Contrazione (in questo contesto contrazione va vista come quando in fisica si parla di entropia in cui una diminuzione del "disordine" di un sistema è associata a una diminuzione di entropia. Quindi con la contrazione del sistema si ha un sistema più coeso, ordinato, coerente) del Sistema Umano.

La contrazione è quello stato in cui l'essere umano è nella perfetta omeostasi tra le varie componenti ulteriori in cui si divide: corpo, mente ed energia. Questo stato si verifica quando la persona svolge un percorso di consapevolezza (più o meno ampio), ed è quindi in uno stato di armonia superiore dovuto alla riduzione degli stati di malessere a seguito della risoluzione delle dinamiche associate. In questo stato d'essere la persona si trova in piena armonia, o comunque in buona armonia e con uno stato di connessione a sentimenti, che gli consento il vivere con calma, serenità, pace, amore e gioia e quindi lontano dallo stress. In questo contesto vive nel distacco emozionale immerso nei sentimenti che ha raggiunto e slegato dalle dinamiche umane.

In questo stato il suo CEM (Campo Elettro Magnetico), è ampio e il suo stato di salute è ottimo.

Questa situazione porta la persona ad avere quindi un equilibrio interiore in ogni sua componente.

Lo stato di malessere è invece definito secondo la TCCU, Stato di Espansione (sempre facendo il parallelo con l'entropia dei sistemi fisici, quando il sistema è in espansione vi è un aumento di entropia e quindi di "Disordine") del Sistema Umano.

Infatti, se il sistema biologico si altera abbiamo una disarmonia tra le componenti corpo, mente ed energia.

Questo significa che come spesso accade, un'emozione ha portato allo sviluppo di una modificazione energetica della persona, ad uno stato di stress che può portare ad un cambio fisiologico. Il perdurare nel tempo di questa alterazione (alterazione del sistema PNEI), provoca una cristallizzazione energetica (collasso d'onda), con conseguente manifestazione nella parte del soma interessato, rendendo stabile la disarmonia. Questa situazione comporta uno sbilanciamento di coscienza/Consapevolezza che porta ad una contrazione del campo elettromagnetico esterno che era entrato nel frattempo in fibrillazione a causa del cambio energetico che era in atto nella persona. Tendenza che si è formalizzata con la cristallizzazione dell'energia. Questo fatto ha portato ad una riduzione di Consapevolezza in quanto la consapevolezza di ciò che ha causato l'alterazione dello stato fisiologico è alla persona non palese altrimenti lo avrebbe evitato. La salute della persona cambia con la riduzione proporzionata del CEM stesso. 
La TB o Tecnica Bioenergetica è il nome scelto per identificare le tecniche e i trattamenti ad approccio Bioenergetico secondo il Metodo SUMMA AUREA®, in ambito Medico e Sanitario e sono lo strumento attraverso cui si può modificare lo stato di Consapevolezza della persona modificandone così il Campo.

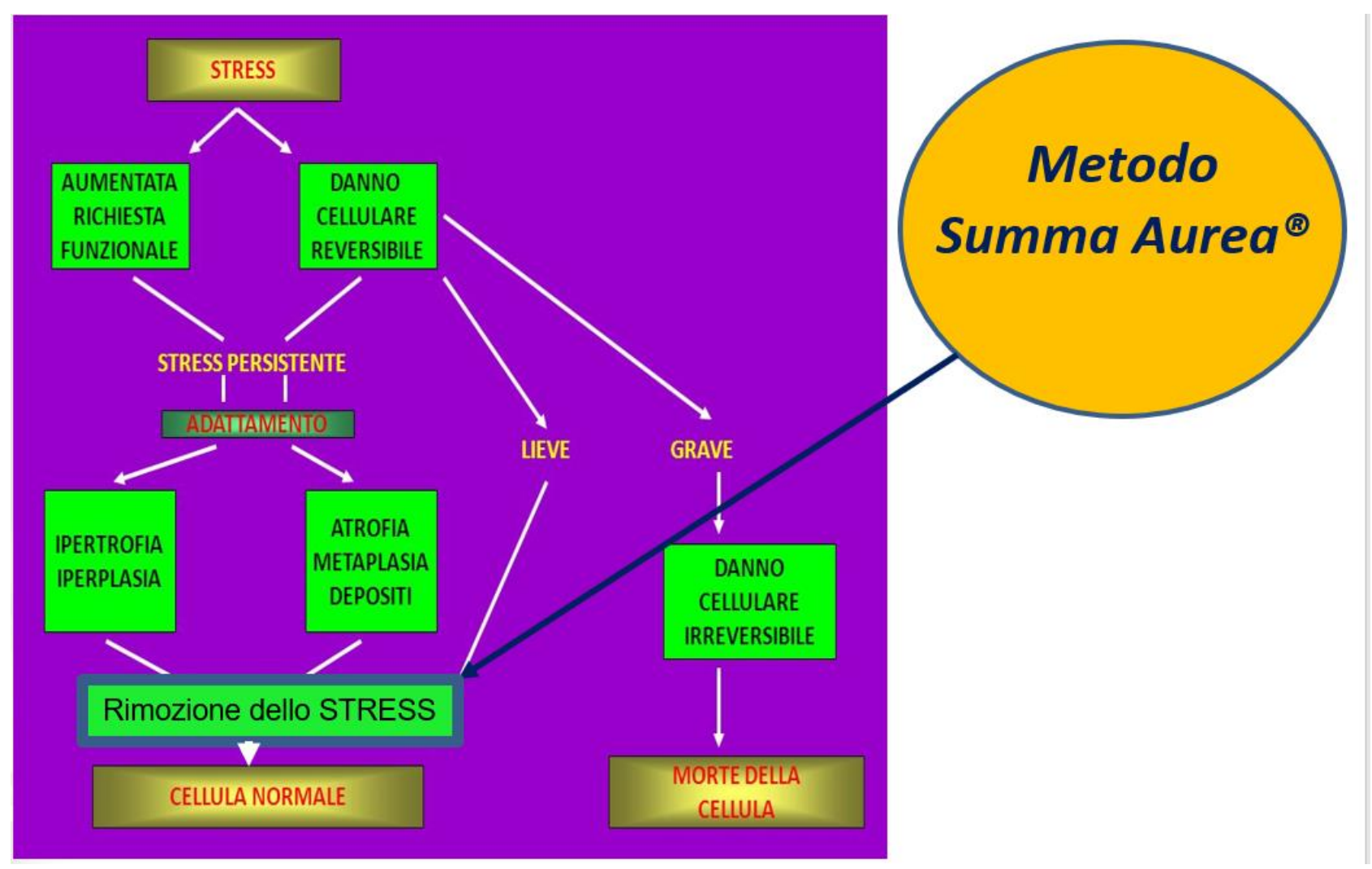

Il Metodo Summa Aurea ${ }^{\circledR}$ è un percorso che consente di far conoscere e sperimentare l'Energia o la Bioenergia che permea l'Universo e ci fornisce la vita che spesso viene comunemente denominata Energia Universale. Questa connessione avviene attraverso l'Intenzione, dato che il Campo Energetico a cui si aggancia questa tecnica è il Vuoto in cui il Campo Scalare ne è il dominatore incontrastato.

In questo contesto in campo possiede cinque principali qualità:

a) indivisibilità, perché non è costituito da parti

b) è di tipo non causale, perché non segue il principio di causalità, concetto legato al principio di indeterminazione della fisica quantistica

c) non può essere sottoposto a processi di tipo riduzionistico

d) non è localizzato in un'unica e precisa dimensione, concetto legato al fenomeno dell'entanglement quantistico

e) è caratterizzato da una totale apertura. 
Il campo, essendo formato da energia, non è statico, ma possiede caratteristiche dinamiche, legate ai processi di evoluzione costante e di interazione continua. La sua dinamicità è di tipo ondulatorio, ritmico ed è specifico per ogni individuo.

Le onde Scalari possono essere riprodotte avvolgendo i fili elettrici formando una figura a forma di otto, in una bobina di Möbius. Quando una corrente elettrica scorre attraverso i fili in direzioni opposte, i campi elettromagnetici opposti dei due fili si annullano a vicenda creando un'onda Scalare. Energia pronta per essere usata, per qualsiasi altra funzione!

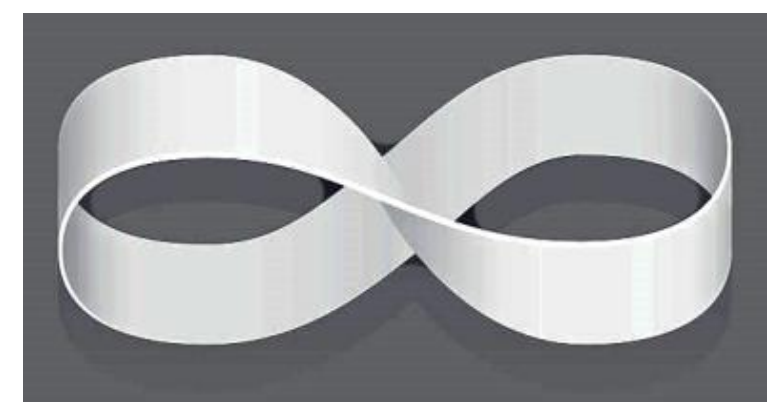

Striscia di Möbius

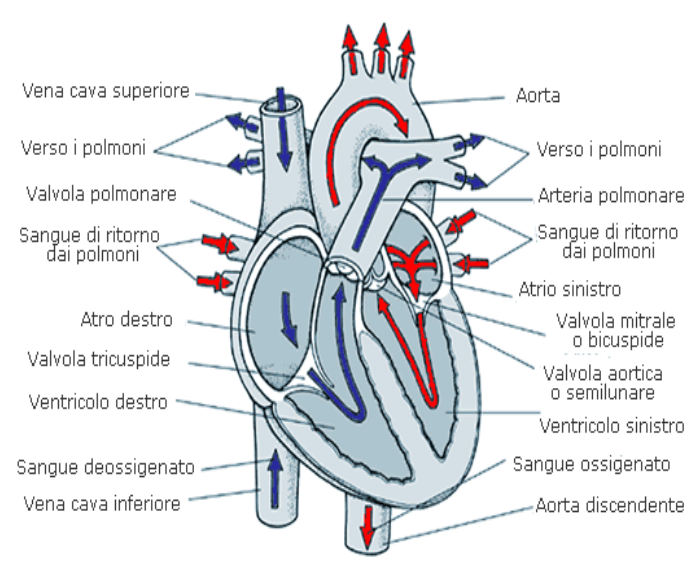

memorizzazione di onde scalari.

In questo contesto diventa rilevante porre l'attenzionenuovamente sul sistema cardiocircolatorio,sul cuore e sul suo flusso sanguigno.

È nel cuore che si trova il centro della bobina vascolare del Möbius: un'intersezione del sangue venoso che passa attraverso l'atrio destro, sovrapponendosi al sangue aortico che passa attraverso l'atrio sinistro.

L'intersezione della bobina di Möbius avviene proprio di fronte ai polmoni, che contengono un'enorme rete di connessioni capillari.

Questa rete capillare funge da batteria per la

Le Ricerche dell'Istituto Hearth Math mostrano che le informazioni sullo stato emotivo di una persona vengono trasmesse in tutto il corpo attraverso il Campo Magnetico del cuore.

I modelli ritmici dei battiti del cuore cambiano in modo significativo, quando sperimentiamo emozioni diverse.

Le emozioni negative, come la rabbia o la frustrazione, come sappiamo, sono associate ad un irregolare e disordinato modello incoerente dei ritmi del cuore, mentre al contrario l'amore, l'amorevolezza, l'apprezzamento sono associati ad una superficie ordinata, creando un modello coerente di attività ritmica del cuore stesso. 
Il cervello del cuore è in grado di registrare direttamente le informazioni del sistema ormonale e di altri sistemi e tradurli in impulsi nervosi, elaborando così direttamente l'informazione che riceve, inviandola poi al cervello lungo un circuito nervoso che utilizza il nervo vago ed i nervi posti ai lati della colonna vertebrale, raggiungendo poi il cervello limbico ed infine la corteccia cerebrale.

Il cervello del cuore può dunque influenzare quello della testa, cioè il nostro modo di pensare, di vedere le cose, la nostra percezione della realtà, le nostre reazioni emozionali: cambio di Consapevolezza.

Attraverso la riarmonizzazione del cuore possiamo lavorare sullo stress che è la principale causa di problemi cardiocircolatori e psicosomatici in generale.

Quello che accade e descriviamo in modo sintetico, è che utilizzando la TB - Tecnica Bioenergetica secondo il Metodo SUMMA AUREA®, rimettiamo in connessione il Campo Scalare del Cuore con il Campo Scalare del Vuoto. È come risintonizzare la radio con la stazione trasmittente recuperando le giuste informazioni, cioè riportare lo stato di Coerenza, prima cardiaca, poi elettromagnetica e infine elettrofisiologica.

Questo comporta, attraverso il meccanismo della Risonanza, che la ritrovata coerenza del Cuore, modifica lo stato del sistema neuro endocrino e la Pineale, i quali possono così rimodulare le loro funzioni come risposta a ciò che il cuore ha comunicato riequilibrando la secernita degli ormoni prodotti direttamente e portando l'organismo in uno stato di equilibrio.

Negli ultimi 10 anni hanno svolto un corso del Metodo Summa Aurea® almeno 1.000 persone e tutte hanno confermato come l'uso delle tecniche disponibili, se utilizzato, modifichi lo stato psicofisiologico, migliorando l'umore, riducendo eventuali dolori e migliorando il rilassamento generale.

È stata cercato conferma strumentale di quello che i corsisti hanno sempre confermato e questo è stato realizzato attraverso una strumentazione basata su HRV.

Il metodo di misurazione di Alpha (il nome dello strumento) viene eseguito con un elettrocardiogramma e analizza quindi la variabilità della frequenza cardiaca. Un ritmo cardiaco ha diversi intervalli. Ogni intervallo è correlato a diversi dipartimenti del sistema nervoso.

L'analisi della relazione tra gli intervalli fornisce informazioni complete su:

\section{- processi di controllo della frequenza cardiaca}

- il sistema nervoso autonomo

- i ritmi dell'attività cerebrale

Essendo tutti i sistemi e gli organi interni del corpo sincronizzati il complesso Alphapuò consentire di rilevare uno stato di malattia prima che compaiano i primi sintomi.

Tutti i sistemi e gli organi del corpo sono interconnessi e sincronizzati e i cambiamenti in un organo o sistema influenzano tutti gli altri organi e sistemi. Con il sistema Alpha (è un dispositivo medico sanitario), si può controllare lo stato funzionale del corpo e la reazione di tutti i sistemi a stress o terapie differenti. 
Il metodo rileva il grado di stress nei sistemi funzionali, o in altre parole, il livello di stress.

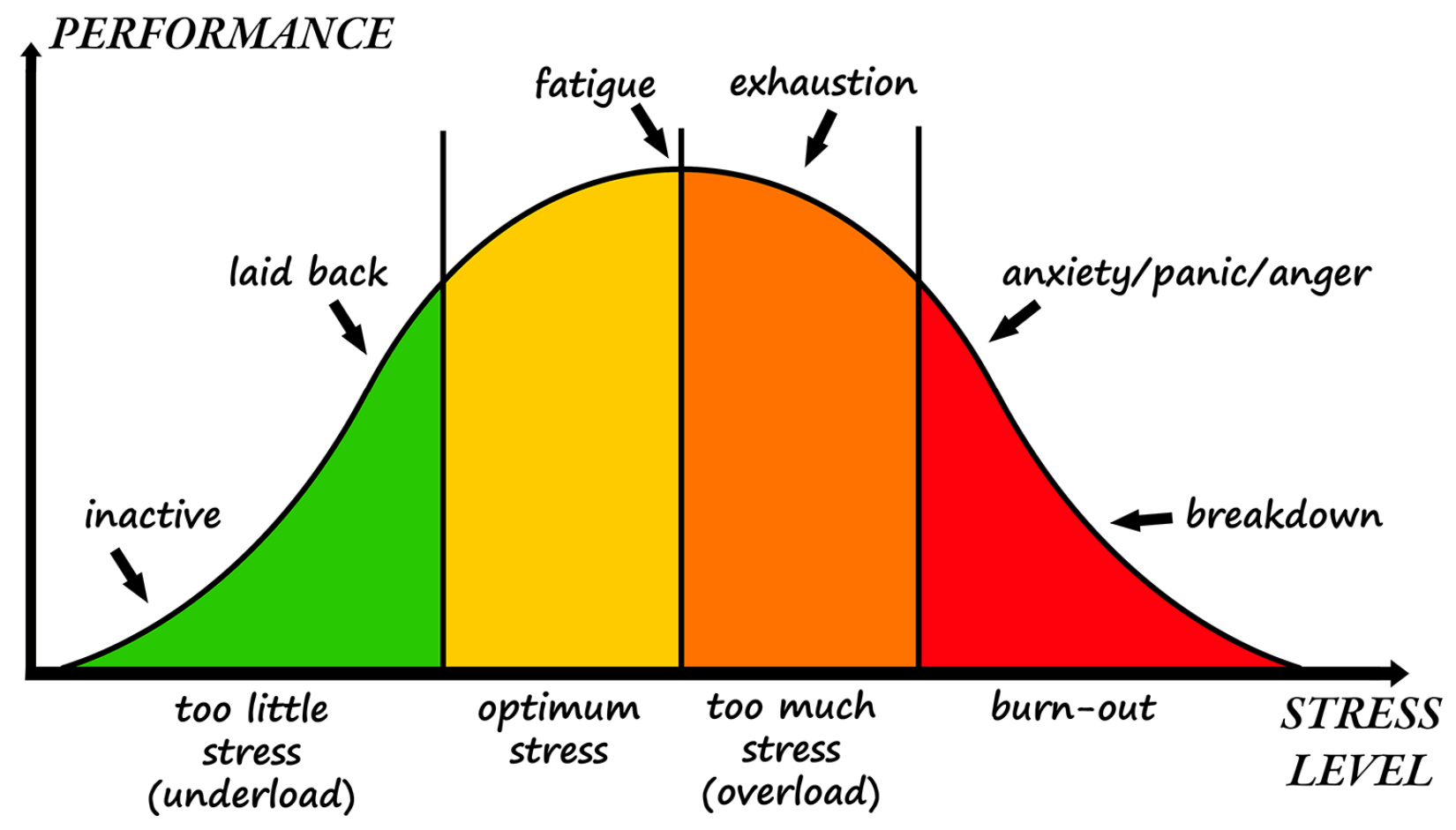


Viene fornito, qui di seguito, lo stato di variazione psicofisico-energetico, di un soggetto che ha ricevuto sia trattamenti diretti sia svolto autotrattamenti attraverso l'uso di Tecniche Bioenergetiche del Metodo Summa Aurea ${ }^{\circledR}$
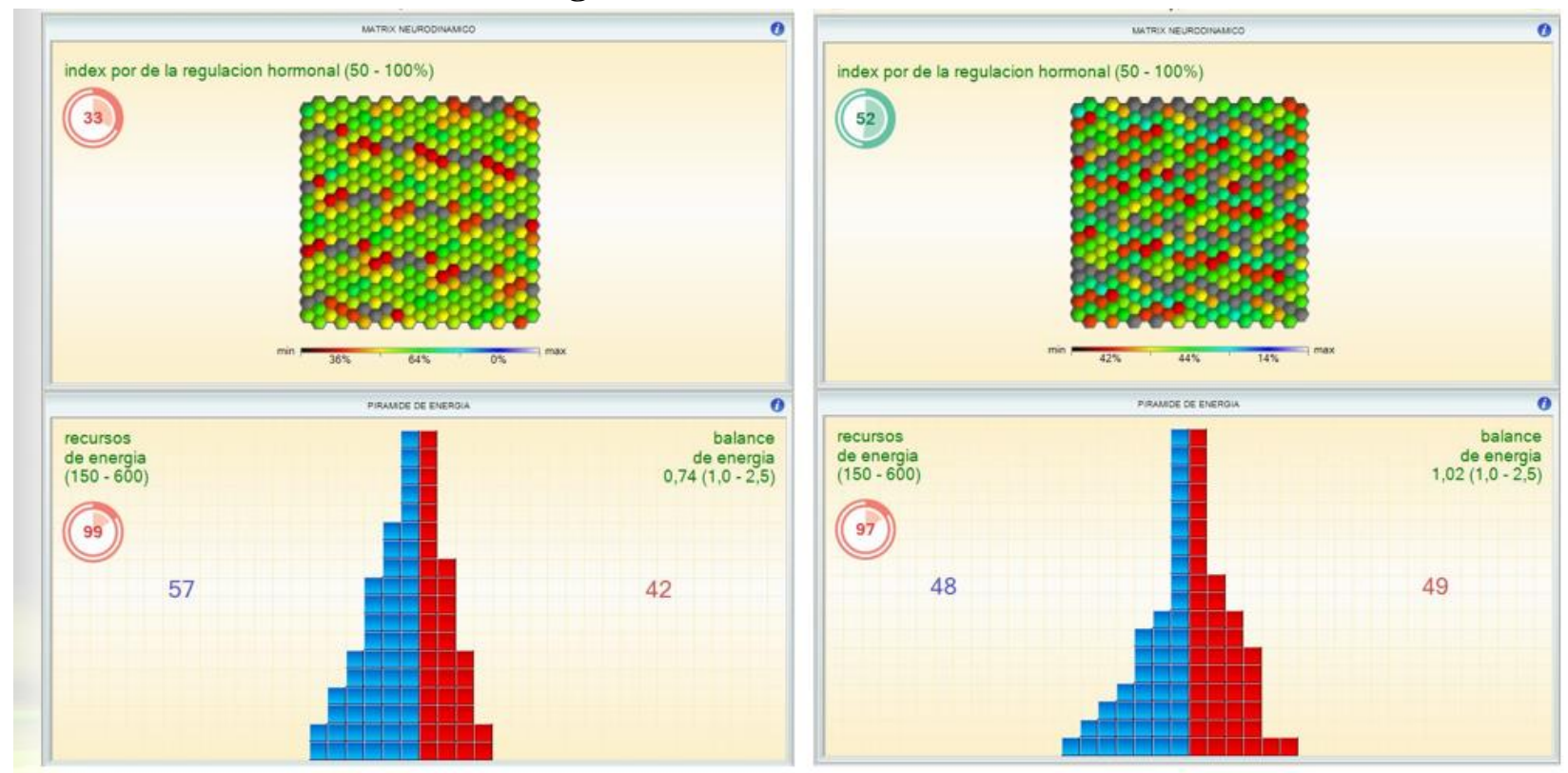

Sistema ormonale a riposo

\section{Effetti sul Sistema ormonale dopo soli 5 minuti di Trattamento Bioenergetico}

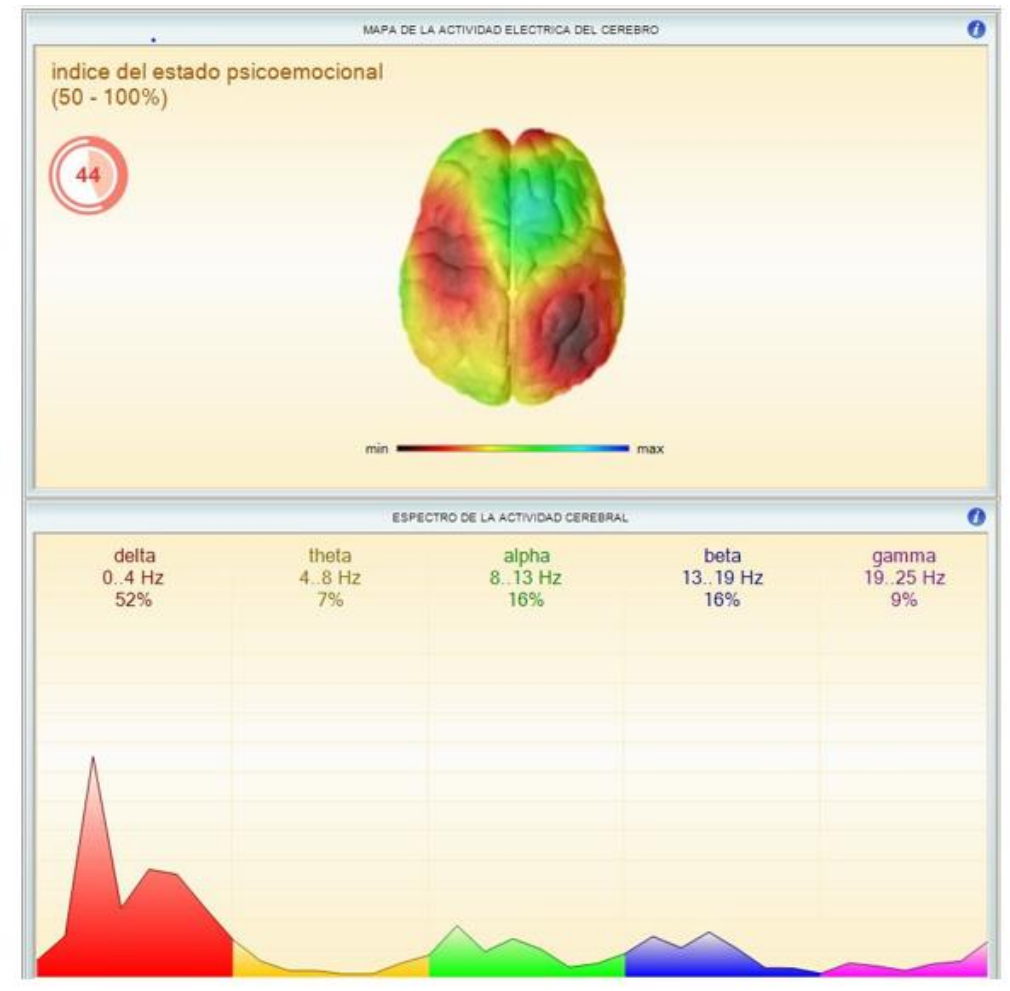

Mappa dell'attività elettrica del cervello 


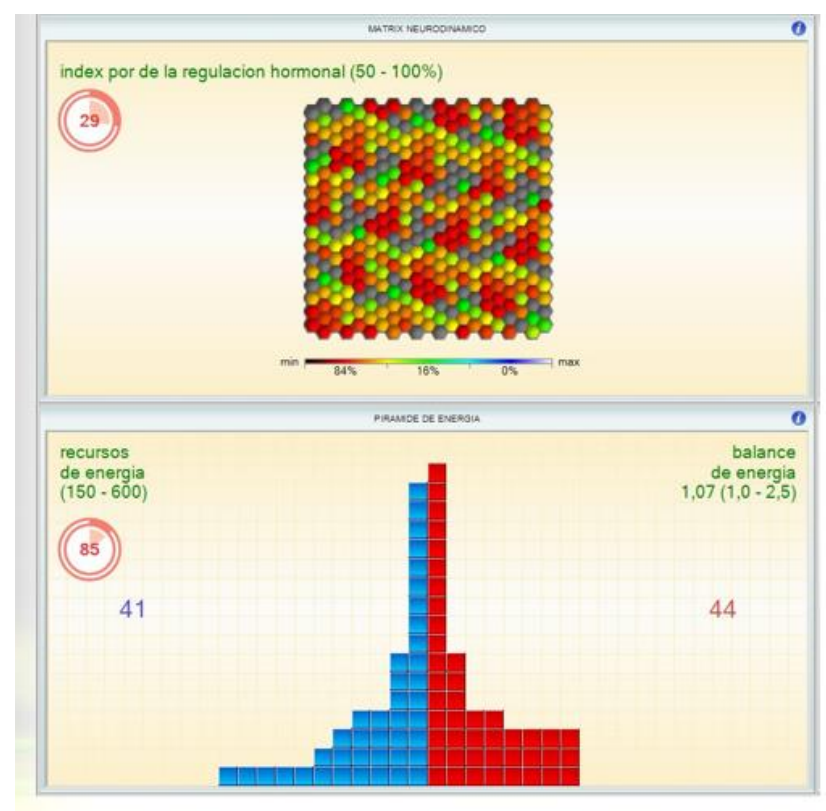

Sistema ormonale a riposo

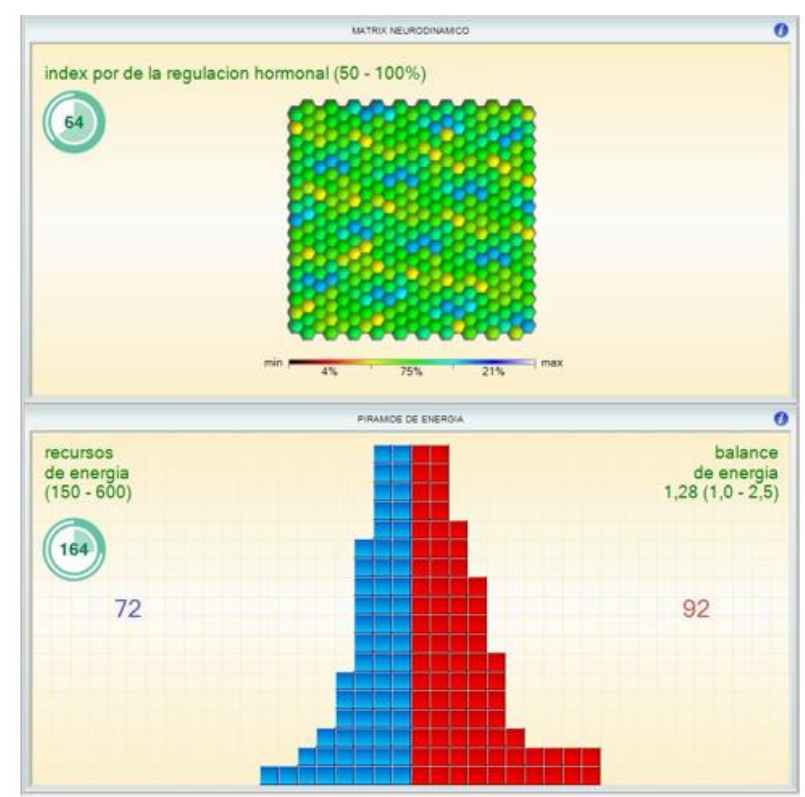

Effetti sul Sistema ormonale dopo soli 30 minuti di Trattamento Bioenergetico

Stessa persona giorno successivo

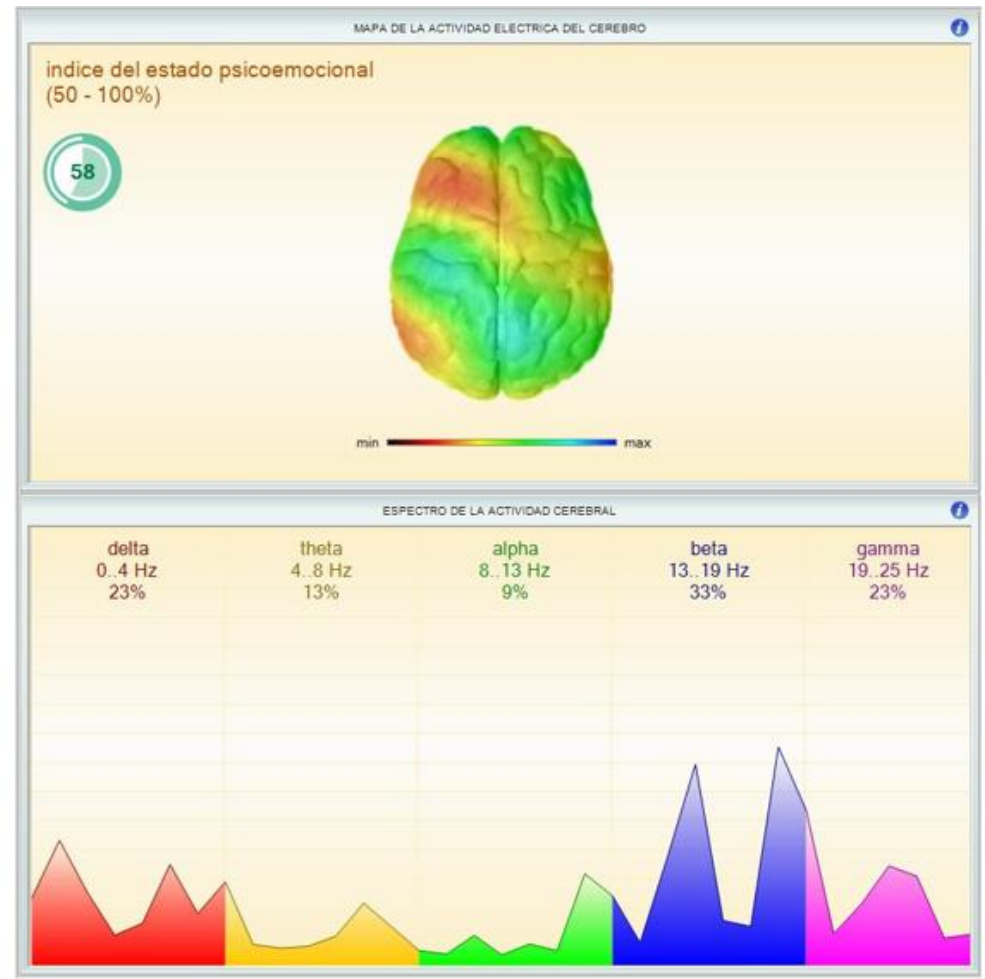

Mappa dell'attività elettrica del cervello 


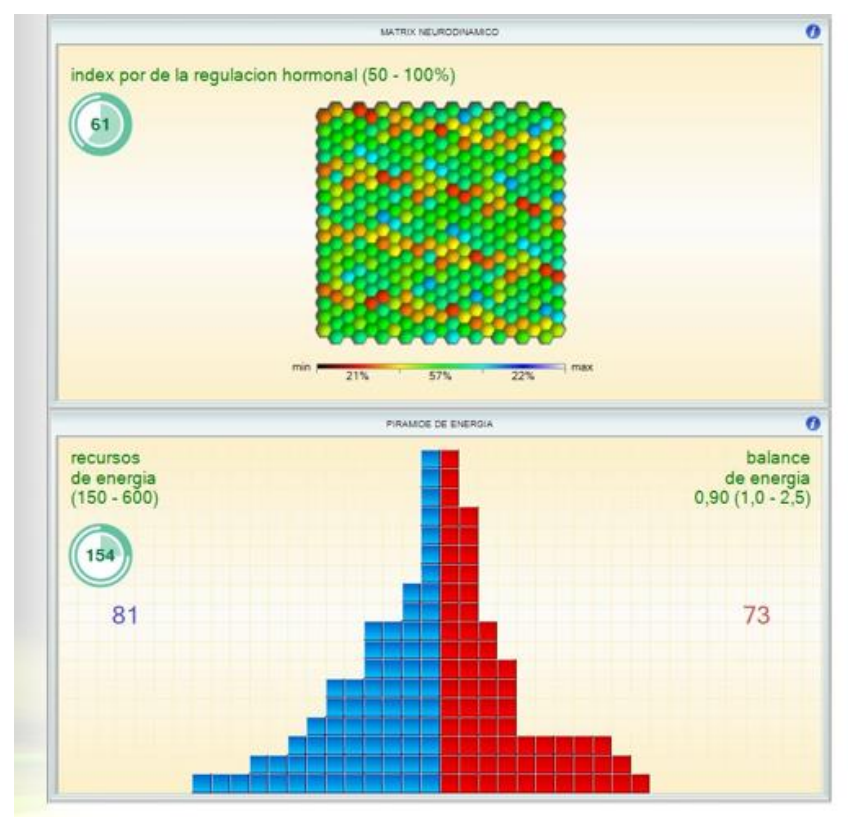

Sistema ormonale a riposo

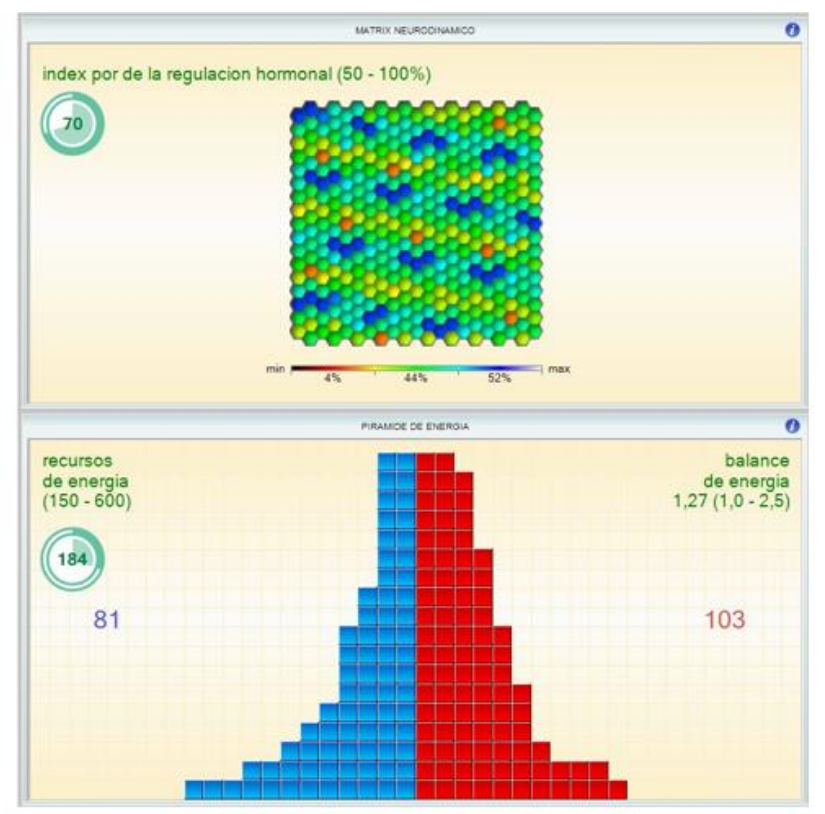

Effetti sul Sistema ormonale dopo soli 5 minuti di AutoTrattamento Bioenergetico

Stessa persona dopo aver fatto il Corso Base Metodo Summa Aurea ${ }^{\circledR}$

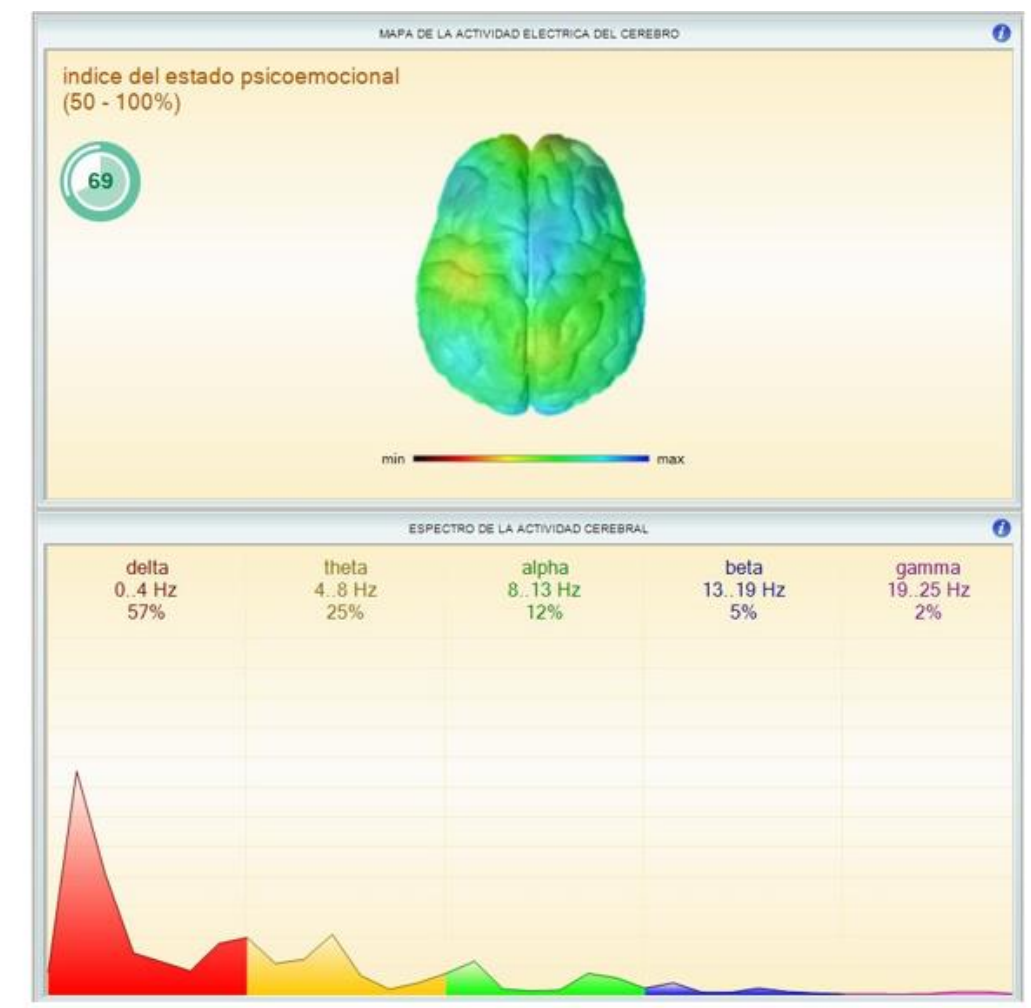




\section{Bibliografia}

1. Lanaro D. Chiera M., Il corpo: dalla costruzione delle emozioni al dolore, 2018 - Franco Angeli Editore

2. Fabbroni R., Resta S.,La Biofisica di un Trattamento energetico. La valenza scientifica delle pratiche ad approccio Bioenergetico, Rivista Scienze Biofisiche (11/2020) DOI: http://dx.medra.org/10.48274/ibil

3. Fabbroni R, Resta S, Sanna A., La paura di ammalarsi: andare incontro a ciò che si voleva evitare,Rivista Scienze Biofisiche (11/2020) DOI:http://dx.medra.org/10.48274/ibi2

4. Fabbroni R., Resta S., Effetti Terapeutici del trattamento sull'infiammazione svolti con la TBTecnica Bioenergetica secondo il Metodo Summa Aurea ${ }^{\circledR}$, Rivista Scienze Biofisiche (11/2020)DOI: https://doi.org/10.48274/IBI3

5. Molinari G., Fabbroni R., Il Principio di Risonanza in un trattamento energetico. Magnetosensing, Rivista Scienze Biofisiche (01/2021) DOI. https://doi.org/10.48274/IBI4

6. Fabbroni R., Aspetti e funzionamento della TB-Tecnica Bioenergetica secondo il Metodo Summa Aurea ${ }^{\circledR}$, Rivista Scienze Biofisiche 1/2021) DOI: https://doi.org/10.48274/IBI5

7. Fabbroni R.,Teoria del Campo di Consapevolezza Unificata e la possibile conferma dell'esistenza dell'Anima, Rivista Scienze Biofisiche (03/2021) DOI: https://doi.org/10.48274/IBI7

8. Argiolas V, Fabbroni R., Trattamento del Dolore Cronico: Emicrania senza aura e Cefalea a grappolo attraverso l'applicazione della TB-Tecnica Bioenergetica secondo il Metodo Summa Aurea $\left.{ }^{\circledR}\right)$, Rivista Scienze Biofisiche (02/2021)DOI: https://doi.org/10.48274/IBI8

9. Calcei E., Fabbroni R.,Cancro al seno: la TB-Tecnica Bioenergetica secondo il Metodo Summa Aurea ${ }^{\circledR}$ come supporto nella Terapia del Dolore e gestione dell'umore, Rivista Scienze Biofisiche (05 /2021) DOI: https://doi.org/10.48274/IBI9

10. Pagnoncelli D., Ledda M.E., Un'esperienza di scambi di trattamenti energetici con la TBTecnica Bioenergetica secondo il Metodo Summa Aurea ${ }^{\circledR}$, Rivista Scienze Biofisiche (02/2021) DOI: https://doi.org/10.48274/IBI10

11. L Suozzo,Sofrologia nella vita quotidiana: Sofrologia, come armonizzare corpo e mente, 2016 